\title{
Direct evaluation of double singular integrals and new free terms in 2D (symmetric) Galerkin BEM
}

\author{
M. Bonnet $^{\mathrm{a}, *}$, M. Guiggiani ${ }^{\mathrm{b}}$ \\ a Laboratoire de Mécanique des Solides (UMR CNRS 7649), Ecole Polytechnique, 91128 Palaiseau cedex, France \\ b Dipartimento di Ingegneria Meccanica, Nucleare e della Produzione, Università di Pisa, via Diotisalvi 2, 56126 Pisa, Italy
}

In this paper a new general algorithm is developed for the direct evaluation of all singular double integrals arising in the 2D Galerkin BEM, including those with hypersingular kernels. A distinguishing feature of the proposed method is that double singular integrals are treated as a whole, that is, not as inner integrals followed by outer ones. Therefore, when applied to the symmetric Galerkin BEM, the proposed technique is strictly symmetry preserving. Moreover, a careful analysis of the limiting process is performed which shows that some new free terms may arise.

Keywords: Symmetric Galerkin BEM; Hypersingular integrals; Free terms; Integration techniques

\section{Introduction}

As is well known, the symmetric Galerkin BEM (SGBEM) typically employs boundary integral equations with hypersingular kernel functions. However, when SGBEM was first formulated [1,2] very little attention was paid to the limiting process which has necessarily to be performed to obtain a boundary integral equation (BIE) with singular kernels. Actually, a proper understanding of the direct treatment of hypersingular boundary integral equations, in the simpler collocation BEM, was obtained only a few years later.

This attitude towards singularities in the SGBEM is still present in the recent review paper [3], whose Section 2 provides a far from complete treatment of the limiting process. To circumvent this sort of difficulties, several indirect regularization techniques have been proposed (and briefly reviewed in Section 8 in [3]). The basic idea is to reduce the order of singularity of the kernel functions thus making the limiting process trivial. In [4] simple solutions were employed, whereas in [5] a procedure based on Stokes theorem (or integration by parts in 2D, as in [6,7]) was developed. Regularization via integration by parts has been

\footnotetext{
${ }^{*}$ Corresponding author. Tel.: +33-1-6933-4129; fax: +33-1-6933-3026.

E-mail addresses: bonnet@1ms.polytechnique.fr (M. Bonnet), guiggiani@ing.unipi.it (M. Guiggiani).
} 
also presented in [8] for Kirchhoff plates. A systematic explanation of the SGBEM and of these regularization techniques can be found in the recent book [9].

Another approach for the evaluation of singular integrals, often called limit to the boundary, was presented for SGBEM by Balakrishna et al. in [10,11]. Recent developments on this technique can be found in [12]. Most of the integrations are performed when the source (singular) point is not on the boundary, thus allowing for the analytical cancellation of all unbounded terms when taking the limit. The procedure is particularly simple for straight elements, but can be extended, with some complications, to curved elements. SGBEM formulations based on the so called finite part integrals have been also developed. In [13,14] they are evaluated numerically, while in [15] an analytical evaluation is pursued.

A method specifically designed to compute integrals with strongly singular kernels for the (unsymmetric) Galerkin BEM in 3D originated in [16] and was enhanced in [17]. It takes advantage of certain symmetry property exhibited by all Cauchy singular kernel functions, even when mapped onto the parameter space. Further details on this approach, but only for strongly singular kernels, can be found in $[18,19]$. To apply it in the SGBEM, that is to hypersingular kernels, an analytic regularization is therefore necessary, as shown in [20] and [21], where simple solutions and Stokes theorem are employed, respectively.

The direct approach [22-27] for the evaluation of element integrals arising from the discretization of strongly singular and hypersingular boundary integral equations is now well established for the collocation BEM and widely used. A fairly comprehensive account of it can be found in [28] (see also [9]).

This paper concerns the extension of the direct approach to hypersingular boundary integral equations in weighted form, like those arising in the SGBEM. The extension is by no means trivial since the Galerkin BEM involves double element integrals. The algorithm presented here deals with both the coincident and adjacent cases, for 2D problems. Indeed it appears that they must be considered together to allow cancellation of potentially unbounded terms.

Double integrals are considered as a whole (i.e. not as inner singular integrals followed by outer nonsingular ones, like in e.g. Ref. [20]) through the introduction of suitable coordinate transformations in the two-dimensional space of intrinsic coordinates. The proposed algorithm is in particular applicable to the symmetric Galerkin BEM (SGBEM) and is devised so as to define in that case a perfectly symmetric integration procedure, even when the numerical quadrature is not exact. In this regard, the present paper also departs from [29] where the direct method was applied to the Galerkin BEM with strongly singular kernels.

In line with previous works on the direct approach, the limiting form of the weighted integral identity must be derived as a small neighbourhood of the singular point vanishes, and this is done, again, after discretization. (Of course, the finite part of divergent integrals is never employed.) Quite surprisingly, it is shown that a new kind of finite free terms arises. It should be noted that, like in the collocation BEM, these free terms are related to the way the limiting process is performed, not to the algorithm eventually adopted for the evaluation of singular integrals. In the present case the limit is carried out on the weighted integral identity. Apparently, an analysis of the free terms arising in the SGBEM based on the vanishing exclusion neighbourhood was so far missing.

\section{Weighted integral statements}

The symmetry of the Galerkin BEM formulation is completely irrelevant in the algorithm that will be developed, the key points being the hypersingularity of the kernels involved and the double integration. However, the proposed technique is strictly symmetry preserving when applied to SGBEM.

We deal with two-dimensional problems associated with linear homogeneous elliptic field equations, among which the Laplace equation $\nabla^{2} \varphi=0$ is the simplest and most common. Let $\Omega$ be a $2 \mathrm{D}$ bounded 
domain with boundary $\Gamma=\partial \Omega$ (possibly with a finite number of corners). By $\boldsymbol{n}(\boldsymbol{x})$ and $\boldsymbol{t}(\boldsymbol{x})$ we indicate, respectively, the normal and tangent unit vectors at the generic point $\boldsymbol{x} \in \Gamma$ (Fig. 1). Cartesian coordinates are used relative to an orthonormal frame $\left(\boldsymbol{e}_{1}, \boldsymbol{e}_{2}\right)$, so that e.g. $\boldsymbol{x}=x_{i} \boldsymbol{e}_{i}$. The starting statement is the classical third Green identity for the density function $\varphi$ and its normal derivative $q=\partial \varphi / \partial n$ on the punctured domain $\Omega_{\varepsilon}=\Omega-v_{\varepsilon}$, with boundary $\Gamma-e_{\varepsilon}+s_{\varepsilon}$ (Fig. 1)

$$
\int_{\Gamma-e_{\varepsilon}+s_{\varepsilon}}[T(\boldsymbol{y}, \boldsymbol{x}) \varphi(\boldsymbol{x})-G(\boldsymbol{y}, \boldsymbol{x}) q(\boldsymbol{x})] \mathrm{d} s_{x}=0,
$$

where $\boldsymbol{y} \in \Gamma$ is the singular point, $G(\boldsymbol{y}, \boldsymbol{x})$ is a fundamental solution and

$$
T(\boldsymbol{y}, \boldsymbol{x})=\frac{\partial G(\boldsymbol{y}, \boldsymbol{x})}{\partial n(\boldsymbol{x})}=\frac{\partial G(\boldsymbol{y}, \boldsymbol{x})}{\partial x_{j}} n_{j}(\boldsymbol{x}) .
$$

As usual, $\varepsilon>0$ is a fixed length that controls the size of $v_{\varepsilon}$ and hence of $s_{\varepsilon}$ and $e_{\varepsilon}$.

If we differentiate $G$ and $T$ with respect to any Cartesian coordinate $y_{i}$ of the singular point and multiply by $n_{i}(\boldsymbol{y})$, we obtain a new pair of (more singular) kernel functions

$$
\begin{aligned}
& \frac{\partial G(\boldsymbol{y}, \boldsymbol{x})}{\partial y_{i}} n_{i}(\boldsymbol{y})=W(\boldsymbol{y}, \boldsymbol{x})=T(\boldsymbol{x}, \boldsymbol{y}), \\
& \frac{\partial T(\boldsymbol{y}, \boldsymbol{x})}{\partial y_{i}} n_{i}(\boldsymbol{y})=V(\boldsymbol{y}, \boldsymbol{x})=V_{i j}(\boldsymbol{y}, \boldsymbol{x}) n_{i}(\boldsymbol{y}) n_{j}(\boldsymbol{x}),
\end{aligned}
$$

which can be combined, exactly like in Eq. (1), in a hypersingular boundary integral identity

$$
\int_{\Gamma-e_{\varepsilon}+s_{\varepsilon}}[V(\boldsymbol{y}, \boldsymbol{x}) \varphi(\boldsymbol{x})-W(\boldsymbol{y}, \boldsymbol{x}) q(\boldsymbol{x})] \mathrm{d} s_{x}=0 .
$$

It should be noted that for the normal $\boldsymbol{n}(\boldsymbol{y})$ to be uniquely defined, the boundary $\Gamma$ at $\boldsymbol{y}$ must be smooth, but this is standard in the Galerkin BEM since $y$ is always located within a boundary element, even when $\Gamma$ has corners.

It is useful to recall that the fundamental solutions are singular at $\boldsymbol{y}=\boldsymbol{x}$

$$
\begin{array}{ll}
G(\boldsymbol{y}, \boldsymbol{x})=\mathrm{O}(\ln r), & T(\boldsymbol{y}, \boldsymbol{x})=\mathrm{O}\left(r^{-1}\right), \\
W(\boldsymbol{y}, \boldsymbol{x})=\mathrm{O}\left(r^{-1}\right), & V(\boldsymbol{y}, \boldsymbol{x})=\mathrm{O}\left(r^{-2}\right),
\end{array}
$$

(where $\boldsymbol{r}=\boldsymbol{x}-\boldsymbol{y}$ and $r=|\boldsymbol{r}|$ ), and that they possess the following symmetry properties:

$$
G(\boldsymbol{y}, \boldsymbol{x})=G(\boldsymbol{x}, \boldsymbol{y}), \quad T(\boldsymbol{y}, \boldsymbol{x})=W(\boldsymbol{x}, \boldsymbol{y}), \quad V(\boldsymbol{y}, \boldsymbol{x})=V(\boldsymbol{x}, \boldsymbol{y}) .
$$

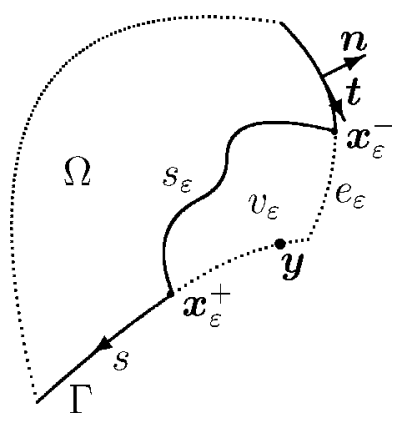

Fig. 1. Exclusion of the singular point $\boldsymbol{y}$ by a vanishing neighbourhood $v_{\varepsilon}$. 
In this paper, the hypersingular kernel $V_{i j}(\boldsymbol{y}, \boldsymbol{x})$ is assumed to have the following, quite general, form:

$$
V_{i j}(\boldsymbol{y}, \boldsymbol{x})=V_{i j}(\boldsymbol{e}, r, \boldsymbol{y})=\frac{1}{r^{2}} \mathscr{V}_{i j}(\boldsymbol{e}, r, \boldsymbol{y}) \quad(\boldsymbol{y} \neq \boldsymbol{x}),
$$

where $\mathscr{V}_{i j}$ is bounded in the limiting case $\boldsymbol{x}=\boldsymbol{y}$, i.e. $r=0$, and the unit vector $\boldsymbol{e}$ is defined as $\boldsymbol{r} / r$. In addition, the symmetry property (6) implies that the nonsingular factor $\mathscr{V}_{i j}(\boldsymbol{e}, r, \boldsymbol{y})$ must satisfy:

$$
\mathscr{V}_{i j}(\boldsymbol{e}, r, \boldsymbol{y})=\mathscr{V}_{j i}(-\boldsymbol{e}, r, \boldsymbol{x}) .
$$

The nonsingular factor $\mathscr{V}_{i j}$ is bounded at $\boldsymbol{x}=\boldsymbol{y}$. If $\boldsymbol{y}$ lies within a region having homogeneous constitutive property (e.g. electrostatic conductivity), the singular behaviour is that of the full-space fundamental solution, e.g.

$$
\mathscr{V}_{i j}(\boldsymbol{e}, 0, \boldsymbol{y})=\frac{1}{2 \pi}\left[\delta_{i j}-2 e_{i} e_{j}\right]
$$

for the Laplace equation. If $\boldsymbol{y}$ lies on a discontinuity line of the constitutive property or a boundary involved in the definition of the fundamental solution, the nonsingular factor takes a different value. For example, the fundamental solution for two half-planes $\Omega^{+}$and $\Omega^{-}$with respective conductivities $k^{+}$and $k^{-}$, bonded at their interface $S=\left\{y_{2}=0\right\}$, is given (for the case $\boldsymbol{x}, \boldsymbol{y} \in \Omega^{+}$) by

$$
V_{i j}(\boldsymbol{y}, \boldsymbol{x})=\frac{k^{+}}{2 \pi}\left\{\frac{1}{r^{2}}\left[\delta_{i j}-2 e_{i} e_{j}\right]+\frac{k^{+}-k^{-}}{k^{+}+k^{-}} \frac{1}{\bar{r}^{2}}\left[\delta_{i 1} \delta_{j 1}-\delta_{i 2} \delta_{j 2}-2\left(\delta_{i 1} \bar{e}_{1}-\delta_{i 2} \bar{e}_{2}\right) \bar{e}_{j}\right]\right\},
$$

where $\overline{\boldsymbol{r}}=\boldsymbol{r}-y_{2} \boldsymbol{e}_{2}, \overline{\boldsymbol{r}}=|\overline{\boldsymbol{r}}|$ and $\overline{\boldsymbol{e}}=\overline{\boldsymbol{r}}$. When $y_{2}=0$, i.e. $\boldsymbol{y} \in S$, the nonsingular factor is therefore given by:

$$
\mathscr{V}_{i j}(\boldsymbol{e}, r, \boldsymbol{y})=\frac{k^{+}}{2 \pi}\left\{\left[\delta_{i j}-2 e_{i} e_{j}\right]+\frac{k^{+}-k^{-}}{k^{+}+k^{-}}\left[\delta_{i 1} \delta_{j 1}-\delta_{i 2} \delta_{j 2}-2\left(\delta_{i 1} \bar{e}_{1} \bar{e}_{j}-\delta_{i 2} \bar{e}_{2}\right) \bar{e}_{j}\right]\right\} .
$$

To facilitate generalizations (e.g. to anisotropic media and elasticity), the nonsingular factor is assumed in this paper to fulfill the requirement

$$
\left.\frac{\partial}{\partial r} \mathscr{V}_{i j}(\boldsymbol{e}, r, \boldsymbol{y})\right|_{r=0}=0 .
$$

Let $\psi(\boldsymbol{y})$ be a function defined on $\Gamma$. Before taking the limit for $\varepsilon \rightarrow 0$, we can use $\psi(\boldsymbol{y})$ to weigh the former integral identities (1) and (4) as typically done in the Galerkin BEM. For the hypersingular identity (4) we have

$$
\int_{\Gamma} \psi(\boldsymbol{y})\left\{\int_{\Gamma-e_{\varepsilon}+s_{\varepsilon}}[V(\boldsymbol{y}, \boldsymbol{x}) \varphi(\boldsymbol{x})-W(\boldsymbol{y}, \boldsymbol{x}) q(\boldsymbol{x})] \mathrm{d} s_{x}\right\} \mathrm{d} s_{y}=0,
$$

or

$$
\int_{\Gamma} \psi(\boldsymbol{y})\left\{\int_{\Gamma-e_{\varepsilon}}[V(\boldsymbol{y}, \boldsymbol{x}) \varphi(\boldsymbol{x})-W(\boldsymbol{y}, \boldsymbol{x}) q(\boldsymbol{x})] \mathrm{d} s_{x}+\int_{s_{\varepsilon}}[V(\boldsymbol{y}, \boldsymbol{x}) \varphi(\boldsymbol{x})-W(\boldsymbol{y}, \boldsymbol{x}) q(\boldsymbol{x})] \mathrm{d} s_{x}\right\} \mathrm{d} s_{y}=0 .
$$

This kind of weighted hypersingular integral identities are useful, for instance, in the symmetric Galerkin BEM [3]. As it will be shown, the algorithm will be developed consistently with the assumption that the external integration precedes the limiting process. 
As customary, the density function $\varphi$ is assumed to be $C^{(1, \alpha)}$ at $\boldsymbol{y}$, that is:

$$
\varphi(\boldsymbol{x})=\varphi(\boldsymbol{y})+\boldsymbol{\nabla} \varphi(\boldsymbol{y}) \cdot(\boldsymbol{x}-\boldsymbol{y})+\mathrm{O}\left(r^{1+\alpha}\right), \quad \boldsymbol{\nabla} \varphi(\boldsymbol{x})=\boldsymbol{\nabla} \varphi(\boldsymbol{y})+\mathrm{O}\left(r^{\alpha}\right), \quad(\alpha>0) .
$$

Through the usual addition and subtraction of the above expansions in the integral on $s_{\varepsilon}$ and rearranging terms in the integral identity (14) we obtain

$$
\begin{aligned}
0=\int_{\Gamma} & \psi(\boldsymbol{y})\left\{\int_{\Gamma-e_{\varepsilon}}[V(\boldsymbol{y}, \boldsymbol{x}) \varphi(\boldsymbol{x})-W(\boldsymbol{y}, \boldsymbol{x}) q(\boldsymbol{x})] \mathrm{d} s_{x}+\varphi(\boldsymbol{y}) \int_{s_{\varepsilon}} V(\boldsymbol{y}, \boldsymbol{x}) \mathrm{d} s_{x}\right. \\
& +\nabla \varphi(\boldsymbol{y}) \cdot\left[\int_{s_{\varepsilon}}\{(\boldsymbol{x}-\boldsymbol{y}) V(\boldsymbol{y}, \boldsymbol{x})-\boldsymbol{n}(\boldsymbol{x}) W(\boldsymbol{y}, \boldsymbol{x})\} \mathrm{d} s_{x}\right] \\
& +\int_{s_{\varepsilon}} V(\boldsymbol{y}, \boldsymbol{x})[\varphi(\boldsymbol{x})-\varphi(\boldsymbol{y})-\nabla \varphi(\boldsymbol{y}) \cdot(\boldsymbol{x}-\boldsymbol{y})] \mathrm{d} s_{x} \\
& \left.-\int_{s_{\varepsilon}} W(\boldsymbol{y}, \boldsymbol{x})[\boldsymbol{\nabla} \varphi(\boldsymbol{x})-\boldsymbol{\nabla} \varphi(\boldsymbol{y})] \cdot \boldsymbol{n}(\boldsymbol{x}) \mathrm{d} s_{x}\right\} \mathrm{d} s_{y},
\end{aligned}
$$

which is more suitable for the subsequent direct evaluation of singular integrals and for the computation of free terms.

We seek the limiting form of the weighted equation (16) as $\varepsilon \rightarrow 0$. In principle any shape may be used for $v_{\varepsilon}$, since the overall result will not depend on this shape. Like for the direct approach in collocation BEM, selecting a circular shape is found to facilitate the analysis and is therefore assumed in the remainder of this paper.

The last two integrals on $s_{\varepsilon}$ in Eq. (16) are $\mathrm{O}\left(\varepsilon^{\alpha}\right)$ by virtue of assumption (15) and thus vanish in the limit for $\varepsilon \rightarrow 0$. As it will be shown in Section 6, the first integral on $s_{\varepsilon}$ in Eq. (16) gives rise to an unbounded $\mathrm{O}\left(\varepsilon^{-1}\right)$ term and, when integrated in the Galerkin BEM, a (somewhat unexpected) bounded free term. The second integral on $s_{\varepsilon}$ in (16) yields a bounded free term $\boldsymbol{c}(\boldsymbol{y})$ in the limit for $\varepsilon \rightarrow 0$ :

$$
\boldsymbol{c}(\boldsymbol{y})=\lim _{\varepsilon \rightarrow 0} \int_{s_{\varepsilon}}\{(\boldsymbol{x}-\boldsymbol{y}) V(\boldsymbol{y}, \boldsymbol{x})-\boldsymbol{n}(\boldsymbol{x}) W(\boldsymbol{y}, \boldsymbol{x})\} \mathrm{d} s_{x},
$$

which occurs also in the direct approach for the collocation BEM in the hypersingular case, see e.g. [24]. The evaluation of $\boldsymbol{c}(\boldsymbol{y})$ is also addressed in Section 6.

Taking into account the above remarks in (16), the direct Galerkin BEM formulation with hypersingular kernels (like required in the SGBEM) is sought as the limiting form as $\varepsilon \rightarrow 0$ of the weighted identity

$$
0=\int_{\Gamma} \psi(\boldsymbol{y})\left\{\boldsymbol{\nabla} \varphi(\boldsymbol{y}) \cdot \boldsymbol{c}(\boldsymbol{y})+\varphi(\boldsymbol{y}) \int_{s_{\varepsilon}} V(\boldsymbol{y}, \boldsymbol{x}) \mathrm{d} s_{x}+\int_{\Gamma-e_{\varepsilon}}\{V(\boldsymbol{y}, \boldsymbol{x}) \varphi(\boldsymbol{x})-W(\boldsymbol{y}, \boldsymbol{x}) q(\boldsymbol{x})\} \mathrm{d} s_{x}\right\} \mathrm{d} s_{y}+\mathrm{O}(\varepsilon),
$$

where $\mathrm{O}(\varepsilon)$ accounts for all vanishing contributions. It should be noted that within curly braces we have precisely the hypersingular boundary integral equation as obtained for the collocation BEM in [23-25], although now the limiting process will wait till after the outer integration is performed. Moreover, the $C^{1, \alpha}$ smoothness assumption for $\varphi$, a standard requirement for the hypersingular collocation BEM, is invoked here mainly to dispose easily of some of the integrals over $s_{\varepsilon}$. Indeed, as a result of the analysis conducted in the following sections, the limiting form as $\varepsilon \rightarrow 0$ of the weighted identity (18) will appear to require only the weaker $C^{0, \alpha}$ smoothness assumption for $\varphi$, which is consistent with other published treatments of the SGBEM.

Up to this point everything is pretty classical. New ideas are introduced in the next sections, towards the goal of developing a technique for the direct evaluation of all singular double integrals (Sections 3-5), along with further treatment of the free terms (Section 6). 


\section{Double integration: preliminary definitions}

Let the boundary $\Gamma$ be modeled by (curvilinear) boundary elements and let $\boldsymbol{y} \in E$ and $\boldsymbol{x} \in E^{\prime}$, with $E$ and $E^{\prime}$ being two such boundary elements. Obviously, this implies that $\Gamma$ is smooth within each element.

In the Galerkin BEM (regardless of its symmetry) we have to deal with double integrals on Cartesian products $E \times E^{\prime}$ of elements. Typically, the weight function $\psi$ has limited support and $E$ belongs to it. If the two elements are disjoint, that is, do not share a common endpoint, the double integration

$$
\int_{E} \int_{E^{\prime}}\{\cdots\} \mathrm{d} s_{x} \mathrm{~d} s_{y}
$$

is performed using ordinary means since the integrand is continuous. On the other hand, singularities in the integrand function arise either when the two elements share one common endpoint (i.e., they are adjacent), or when they are coincident (i.e., $E=E^{\prime}$ ). In these cases, according to the starting identity (18), the appropriate setting is

$$
\int_{E} \int_{E^{\prime}(\boldsymbol{y}, \varepsilon)}\{\cdots\} \mathrm{d} s_{x} \mathrm{~d} s_{y}
$$

where

$$
E^{\prime}(\boldsymbol{y}, \varepsilon)=\left\{\boldsymbol{x} \in E^{\prime}:|\boldsymbol{x}-\boldsymbol{y}| \geqslant \varepsilon, \quad \text { with } \boldsymbol{y} \in E\right\}=E^{\prime} \backslash e_{\varepsilon}(\boldsymbol{y}) .
$$

In fact, one has $E^{\prime}(\boldsymbol{y}, \varepsilon)=E^{\prime}$ in the disjoint case (19) and for sufficiently small $\varepsilon$, which is thus included in this setting.

Each geometric boundary element is analytically defined by means of (usually polynomial) parametric equations. A point $\boldsymbol{x}$ of $E^{\prime}$ is typically given by

$$
\boldsymbol{x}=\boldsymbol{x}(\xi)=\sum_{p=1}^{N_{e}} N_{p}(\xi) \tilde{\boldsymbol{x}}^{p}
$$

where $\xi \in[-1,1]$ is the parameter (or intrinsic) coordinate, $N_{p}(\xi)$ are cardinal shape functions and $\tilde{\boldsymbol{x}}$ are the geometric nodes of $E^{\prime}$. Of course, the use of cardinal shape function is just a matter of practical convenience. Any set of parametric equations for $\boldsymbol{x}(\xi)$ would fit the purpose.

We will denote by $\boldsymbol{a}(\xi)$ and $\boldsymbol{t}(\xi)$, respectively, the natural and unit tangent vector to the element $E^{\prime}$ at $\boldsymbol{x}(\xi)$ :

$$
\boldsymbol{a}(\xi)=\frac{\mathrm{d} \boldsymbol{x}}{\mathrm{d} \xi}=\sum_{p=1}^{N_{e}} \frac{\mathrm{d} N_{p}}{\mathrm{~d} \xi}(\xi) \tilde{\boldsymbol{x}}^{p}, \quad \text { so that } \quad \boldsymbol{t}(\xi)=\frac{\boldsymbol{a}(\xi)}{|\boldsymbol{a}(\xi)|}=\frac{\boldsymbol{a}(\xi)}{a(\xi)},
$$

and by $\boldsymbol{m}(\xi)$ and $\boldsymbol{n}(\xi)$ the natural and unit normal vectors:

$$
\boldsymbol{m}(\xi)=\left(\boldsymbol{e}_{1} \wedge \boldsymbol{e}_{2}\right) \wedge \boldsymbol{a}(\xi), \quad \boldsymbol{n}(\xi)=\frac{\boldsymbol{m}(\xi)}{|\boldsymbol{m}(\xi)|}=\frac{\boldsymbol{m}(\xi)}{a(\xi)}
$$

In terms of components of $\boldsymbol{m}$ and $\boldsymbol{a}$ we have that

$$
m_{1}=-a_{2}, \quad m_{2}=a_{1} .
$$

Moreover, it should be noted that $a(\xi)=|\boldsymbol{a}(\xi)|$ is the Jacobian. In fact

$$
\boldsymbol{n}(\boldsymbol{x}) \mathrm{d} s_{x}=\boldsymbol{n}(\xi) a(\xi) \mathrm{d} \xi=\boldsymbol{m}(\xi) \mathrm{d} \xi,
$$


where, for simplicity, we adopted the slightly sloppy notation $\boldsymbol{n}(\xi)$ to mean $\boldsymbol{n}(\boldsymbol{x}(\xi))$. We also define

$$
\boldsymbol{b}(\xi)=\frac{\mathrm{d} \boldsymbol{a}}{\mathrm{d} \xi}=\frac{\mathrm{d}^{2} \boldsymbol{x}}{\mathrm{d} \xi^{2}}=\sum_{p=1}^{N_{e}} \frac{\mathrm{d}^{2} N_{p}}{\mathrm{~d} \xi^{2}}(\xi) \tilde{\boldsymbol{x}}^{p} .
$$

Similarly to Eq. (22), we have for the singular point $\boldsymbol{y} \in E$

$$
\boldsymbol{y}=\boldsymbol{y}(\eta)=\sum_{p=1}^{N_{e}} N_{p}(\eta) \tilde{\boldsymbol{y}}^{p},
$$

with $-1 \leqslant \eta \leqslant 1$. Obviously, $\tilde{\boldsymbol{y}}^{p}$ are the geometric nodes of $E$. In general, the distance vector $\boldsymbol{r}$ between the points $\boldsymbol{x}$ and $\boldsymbol{y}$ is therefore given by

$$
\boldsymbol{r}=\boldsymbol{x}(\xi)-\boldsymbol{y}(\eta)=\sum_{p=1}^{N_{e}}\left[N_{p}(\xi) \tilde{\boldsymbol{x}}^{p}-N_{p}(\eta) \tilde{\boldsymbol{y}}^{p}\right]
$$

and has modulus $r=|\boldsymbol{r}|$.

\section{Double integration over coincident elements}

Let $\boldsymbol{x}$ and $\boldsymbol{y}$ belong to the same boundary element $E^{\prime}=E$. We will consider the integral with hypersingular kernel (the strongly singular one is just simpler). According to (20) we have

$$
I_{C}=\int_{E} \int_{E(\boldsymbol{y}, \varepsilon)} V_{i j}(\boldsymbol{y}, \boldsymbol{x}) n_{i}(\boldsymbol{y}) n_{j}(\boldsymbol{x}) \psi(\boldsymbol{y}) \varphi(\boldsymbol{x}) \mathrm{d} s_{x} \mathrm{~d} s_{y}
$$

where $E(\boldsymbol{y}, \varepsilon)$ is defined by (21) with $E^{\prime}=E$. Expression (28) for the distance vector $\boldsymbol{r}$ becomes in this case

$$
\boldsymbol{r}=\boldsymbol{x}(\xi)-\boldsymbol{y}(\eta)=\sum_{p=1}^{N_{e}}\left[N_{p}(\xi)-N_{p}(\eta)\right] \tilde{\boldsymbol{y}}^{p}
$$

and $(r=0) \Longleftrightarrow(\boldsymbol{x}=\boldsymbol{y}) \Longleftrightarrow(\xi=\eta)$. Therefore, in the parameter space we have to integrate over the square $(\eta, \xi) \in[-1,1] \times[-1,1]$ minus a (narrow, non uniform) strip across the $\xi=\eta$ diagonal, as shown in Fig. 2. The strip is the image of $E \times e_{\varepsilon}(\boldsymbol{y})$.

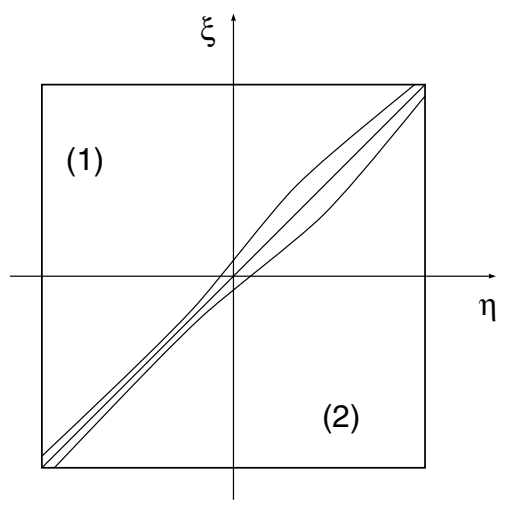

Fig. 2. Coincident case: integration domain and exclusion vanishing strip in the parameter space. 


\subsection{Coordinate transformations and expansions}

The double integration (29) will be treated by subdividing the square $(\eta, \xi) \in[-1,1] \times[-1,1]$ in the parameter space into two triangular regions, labeled (1) and (2) as shown in Fig. 2. In each region a new pair of coordinates $(u, v)$ will be introduced according to the following scheme

$$
\begin{aligned}
& \text { region (1): }\left\{\begin{array}{l}
\eta=u-(1+u) v=\eta^{(1)}(u, v), \\
\xi=u+(1-u) v=\xi^{(1)}(u, v),
\end{array}\right. \\
& \text { region (2): }\left\{\begin{array}{l}
\eta=u+(1-u) v=\eta^{(2)}(u, v), \\
\xi=u-(1+u) v=\xi^{(2)}(u, v),
\end{array}\right.
\end{aligned}
$$

with, in both regions, $u \in[-1,1]$ and $v \in[0,1]$. In some cases the notation $\xi(u, v)$ and $\eta(u, v)$ will be used to refer to (31) and (32).

It should be noted that in both cases if $v=0$ we have $\xi=\eta$, that is, more precisely

$$
\xi^{(i)}(u, 0)=\eta^{(i)}(u, 0)=u .
$$

Therefore, $v=0$ activates the singularity. Another feature of these coordinate transformations is that $u$ and $v$ vary between fixed values, that is the range of variation of each coordinate does not depend on the other one. Also useful is the relation

$$
\mathrm{d} \xi \mathrm{d} \eta=2(1-v) \mathrm{d} u \mathrm{~d} v .
$$

In terms of the new coordinates $u$ and $v$, the distance vector $\boldsymbol{r}$ defined in (30) becomes

$$
\boldsymbol{r}=\boldsymbol{r}^{(i)}(u, v)=\boldsymbol{x}\left(\xi^{(i)}(u, v)\right)-\boldsymbol{y}\left(\eta^{(i)}(u, v)\right)=\sum_{p=1}^{N_{e}}\left[N_{p}\left(\xi^{(i)}(u, v)\right)-N_{p}\left(\eta^{(i)}(u, v)\right)\right] \tilde{\boldsymbol{y}}^{p} .
$$

We are interested in the Taylor expansion of functions $\boldsymbol{r}^{(i)}(u, v)$ as a function of $v$ and near $v=0$. It is a simple matter to obtain from Eq. (30) the following more explicit expressions for the derivatives of $\boldsymbol{r}$ appearing in (37)

$$
\begin{aligned}
&\left.\frac{\partial \boldsymbol{r}^{(i)}}{\partial v}\right|_{v=0}=\left[\frac{\mathrm{d} \boldsymbol{x}}{\mathrm{d} \xi} \frac{\partial \xi^{(i)}}{\partial v}-\frac{\mathrm{d} \boldsymbol{y}}{\mathrm{d} \eta} \frac{\partial \eta^{(i)}}{\partial v}\right]_{v=0}=\sum_{p=1}^{N_{e}}\left[\frac{\mathrm{d} N_{p}}{\mathrm{~d} u}(u)( \pm 1-u)-\frac{\mathrm{d} N_{p}}{\mathrm{~d} u}(u)(\mp 1-u)\right] \tilde{\boldsymbol{y}}^{p} \\
&= \pm 2 \sum_{p=1}^{N_{e}} \frac{\mathrm{d} N_{p}}{\mathrm{~d} u}(u) \tilde{\boldsymbol{y}}^{p}= \pm 2 \boldsymbol{a}(u), \\
&\left.\frac{\partial^{2} \boldsymbol{r}^{(i)}}{\partial v^{2}}\right|_{v=0}=\sum_{p=1}^{N_{e}}\left[\frac{\mathrm{d}^{2} N_{p}}{\mathrm{~d} u^{2}}(u)( \pm 1-u)^{2}-\frac{\mathrm{d}^{2} N_{p}}{\mathrm{~d} u^{2}}(u)(\mp 1-u)^{2}\right] \tilde{\boldsymbol{y}}^{p}=\mp 4 u \sum_{p=1}^{N_{e}} \frac{\mathrm{d}^{2} N_{p}}{\mathrm{~d} u^{2}}(u) \tilde{\boldsymbol{y}}^{p}=\mp 4 u \boldsymbol{b}(u),
\end{aligned}
$$

where, since $u=\xi=\eta$ when $v=0, \boldsymbol{a}$ and $\boldsymbol{b}$ are precisely the functions defined in Eqs. (23) and (26) (with just $u$ replacing $\xi)$. The Taylor expansion of $\boldsymbol{r}^{(i)}(u, v)$ readily follows:

$$
\begin{aligned}
\boldsymbol{r}^{(i)}(u, v) & =\boldsymbol{r}^{(i)}(u, 0)+\left.\frac{\partial \boldsymbol{r}^{(i)}}{\partial v}\right|_{v=0} v+\left.\frac{1}{2} \frac{\partial^{2} \boldsymbol{r}^{(i)}}{\partial v^{2}}\right|_{v=0} v^{2}+\mathrm{O}\left(v^{3}\right)=0 \pm 2 \boldsymbol{a}(u) v \mp 2 u \boldsymbol{b}(u) v^{2}+\mathrm{O}\left(v^{3}\right) \\
& = \pm 2 v\left[\boldsymbol{a}(u)-u v \boldsymbol{b}(u)+\mathrm{O}\left(v^{2}\right)\right]=v \hat{\boldsymbol{r}}^{(i)}(u, v) .
\end{aligned}
$$

The last row defines a continuous function $\hat{\boldsymbol{r}}^{(i)}$

$$
\hat{\boldsymbol{r}}^{(i)}(u, v)= \pm 2 \boldsymbol{a}(u) \mp 2 u v \boldsymbol{b}(u)+\mathrm{O}\left(v^{2}\right)
$$


with the property that $\hat{\boldsymbol{r}}^{(i)}(u, 0)= \pm 2 \boldsymbol{a}(u) \neq 0$. Similar definitions (with \pm or $\mp$ ) will be used throughout the paper, where the first sign refers to region (1), and the second one to region (2) (Fig. 2).

Similarly, from (38), the distance $r=|\boldsymbol{r}|$ has, the following Taylor expansion

$$
r=\sqrt{\boldsymbol{r} \cdot \boldsymbol{r}}=2 v\left[a(u)-u v \frac{\mathrm{d} a}{\mathrm{~d} u}(u)+\mathrm{O}\left(v^{2}\right)\right]=v \hat{r}(u, v)
$$

where $a(u)$ is defined by (23) and $\mathrm{d} a / \mathrm{d} u=(\boldsymbol{a}(u) \cdot \boldsymbol{b}(u)) / a(u)$, and having set

$$
\hat{r}(u, v)=|\hat{\boldsymbol{r}}(u, v)|=2 a(u)\left[1-\frac{u v}{a(u)} \frac{\mathrm{d} a}{\mathrm{~d} u}(u)+\mathrm{O}\left(v^{2}\right)\right],
$$

while the unit position vector $\boldsymbol{e}$ is such that:

$$
\boldsymbol{e}^{(i)}(u, v)=\frac{\hat{\boldsymbol{r}}^{(i)}(u, v)}{\hat{r}(u, v)}=\boldsymbol{t}(u)+\mathrm{O}(v) .
$$

The integrand function in Eq. (29), as a function of $u$ and $v$, becomes

$$
\begin{aligned}
V_{i j}(\boldsymbol{y}, \boldsymbol{x}) n_{i}(\boldsymbol{y}) n_{j}(\boldsymbol{x}) \psi(\boldsymbol{y}) \varphi(\boldsymbol{x}) \mathrm{d} s_{x} \mathrm{~d} s_{y}= & \frac{1}{r^{2}} \mathscr{V}_{i j}(\boldsymbol{e}, r, \boldsymbol{y}) m_{i}(\eta) m_{j}(\xi) \psi(\eta) \varphi(\xi) \mathrm{d} \xi \mathrm{d} \eta \\
= & \frac{1}{v^{2} \hat{r}^{2}(u, v)} \mathscr{V}_{i j}\left(\boldsymbol{e}^{(i)}(u, v), r(u, v), \boldsymbol{y}\left(\eta^{(i)}(u, v)\right)\right) m_{i}\left(\eta^{(i)}(u, v)\right) m_{j} \\
& \times\left(\xi^{(i)}(u, v)\right) \psi\left(\eta^{(i)}(u, v)\right) \varphi\left(\xi^{(i)}(u, v)\right) 2(1-v) \mathrm{d} u \mathrm{~d} v \\
= & \frac{1}{v^{2}} \mathscr{F}\left(\xi^{(i)}(u, v), \eta^{(i)}(u, v)\right)(1-v) \mathrm{d} u \mathrm{~d} v=\frac{1}{v^{2}} F^{(i)}(u, v) \mathrm{d} u \mathrm{~d} v .
\end{aligned}
$$

This expression also defines the nonsingular functions $F^{(i)}(u, v)$ and $\mathscr{F}(\xi, \eta)$ (note that $\mathscr{F}$ is the same on regions (1) and (2)). Moreover, $\psi(\eta)$ means $\psi(\boldsymbol{y}(\eta))$ and $\varphi(\xi)$ means $\varphi(\boldsymbol{x}(\xi))$.

The direct algorithm for the evaluation of hypersingular integrals (see, e.g., [28] for a comprehensive description for the collocation BEM) relies on a two-term Taylor expansion of $F(u, v)$ around $v=0$

$$
F(u, v)=F(u, 0)+\frac{\partial F}{\partial v}(u, 0) v+\mathrm{O}\left(v^{2}\right) .
$$

Here, one observes from the definition (42) of $F(u, v)$ and $\mathscr{F}(\xi, \eta)$ that

$$
F^{(i)}(u, 0)=\mathscr{F}\left(\xi^{(i)}(u, 0), \eta^{(i)}(u, 0)\right)=\mathscr{F}(u, u)=\frac{1}{2} \mathscr{V}_{i j}(\boldsymbol{t}(u), 0, \boldsymbol{y}(u)) n_{i}(u) n_{j}(u) \psi(u) \varphi(u)
$$

and also that

$$
\begin{aligned}
\frac{\partial F^{(i)}}{\partial v} & \left.=\frac{\partial \mathscr{F}}{\partial \xi} \frac{\partial \xi^{(i)}}{\partial v}+\frac{\partial \mathscr{F}}{\partial \eta} \frac{\partial \eta^{(i)}}{\partial v}\right)(1-v)-\mathscr{F}\left(\xi^{(i)}(u, v), \eta^{(i)}(u, v)\right) \\
& =\left(\frac{\partial \mathscr{F}}{\partial \xi}( \pm 1-u)+\frac{\partial \mathscr{F}}{\partial \eta}(\mp 1-u)\right)(1-v)-\mathscr{F}\left(\xi^{(i)}(u, v), \eta^{(i)}(u, v)\right),
\end{aligned}
$$

which means that

$$
\frac{\partial F^{(1)}}{\partial v}+\frac{\partial F^{(2)}}{\partial v}=-2 u\left(\frac{\partial \mathscr{F}}{\partial \xi}+\frac{\mathscr{F}}{\partial \eta}\right)(1-v)-\left[\mathscr{F}\left(\xi^{(1)}, \eta^{(1)}\right)+\mathscr{F}\left(\xi^{(2)}, \eta^{(2)}\right)\right] .
$$


If this expression is evaluated at $v=0$, that is at $\xi=\eta=u$, we obtain

$$
\left[\frac{\partial F^{(1)}}{\partial v}+\frac{\partial F^{(2)}}{\partial v}\right]_{v=0}=-2 u \frac{\mathrm{d}}{\mathrm{d} u}(\mathscr{F}(u, u))-2 \mathscr{F}(u, u)=-2 \frac{\mathrm{d}}{\mathrm{d} u}(u \mathscr{F}(u, u))=-2 \frac{\mathrm{d}}{\mathrm{d} u}(u F(u, 0)),
$$

where the last step is based on (44). This expression will later prove very useful.

Once the expansion of the singular integrand function has been obtained, we have to consider, as usual in the direct approach, the image in the parameter plane of the exclusion vanishing neighbourhood $e_{\varepsilon}(\boldsymbol{y})$ (Fig. 1). The boundary of $e_{\varepsilon}(\boldsymbol{y})$ is defined by the condition

$$
r=|\boldsymbol{x}(\xi(u, v))-\boldsymbol{y}(\eta(u, v))|=\varepsilon .
$$

This condition, together with expansion (39) for $r(u, v)$, leads, on each triangular subregion (Fig. 2), to

$$
\varepsilon=r(u, v)=v \hat{r}(u, v)=2 v a(u)\left[1-\frac{u v}{a(u)} \frac{\mathrm{d} a}{\mathrm{~d} u}(u)+\mathrm{O}\left(v^{2}\right)\right],
$$

which, upon reversion, defines the function

$$
v_{\varepsilon}=\alpha(\varepsilon, u)=\frac{\varepsilon}{2 a(u)}\left[1+\frac{u}{2 a^{2}(u)} \frac{\mathrm{d} a}{\mathrm{~d} u}(u) \varepsilon+\mathrm{O}\left(\varepsilon^{2}\right)\right] .
$$

The function $\alpha(\varepsilon, u)$ provides, for any given values of $u$ and $\varepsilon$, the value of $v$ corresponding to the boundary of the exclusion strip in Fig. 2.

\subsection{Double singular integrals in parametric coordinates}

According to Eqs. (42) and (48), the hypersingular double integral (29) can now be expressed in terms of the parametric coordinates $u$ and $v$

$$
I_{C}=\int_{E} \int_{E(\boldsymbol{y}, \varepsilon)} V_{i j}(\boldsymbol{y}, \boldsymbol{x}) n_{i}(\boldsymbol{y}) n_{j}(\boldsymbol{x}) \psi(\boldsymbol{y}) \varphi(\boldsymbol{x}) \mathrm{d} s_{x} \mathrm{~d} s_{y}=\int_{-1}^{1}\left\{\int_{\alpha(\varepsilon, u)}^{1} \frac{1}{v^{2}}\left[F^{(1)}(u, v)+F^{(2)}(u, v)\right] \mathrm{d} v\right\} \mathrm{d} u .
$$

It should be observed that this is an exact restatement of the original integral over $E \times E(\boldsymbol{y}, \varepsilon)$, with constant $\varepsilon$, in terms of a new pair of parametric coordinates. Of course, care has been taken to preserve the limiting process.

\subsection{The direct approach for coincident elements}

Following the direct algorithm for the evaluation of hypersingular integrals, the first two terms of expansion (43) are added and subtracted in (49) thus obtaining

$$
I_{C}=I_{0}+I_{1}+I_{2}
$$

having put

$$
\begin{aligned}
& I_{0}=\int_{-1}^{1}\left\{\int_{\alpha(\varepsilon, u)}^{1}\left[F^{(1)}(u, v)+F^{(2)}(u, v)-2 F(u, 0)-\left(\frac{\partial F^{(1)}}{\partial v}(u, 0)+\frac{\partial F^{(2)}}{\partial v}(u, 0)\right) v\right] \frac{\mathrm{d} v}{v^{2}}\right\} \mathrm{d} u, \\
& I_{1}=\int_{-1}^{1}\left(\frac{\partial F^{(1)}}{\partial v}(u, 0)+\frac{\partial F^{(2)}}{\partial v}(u, 0)\right) \int_{\alpha(\varepsilon, u)}^{1} \frac{\mathrm{d} v}{v} \mathrm{~d} u, \\
& I_{2}=\int_{-1}^{1} 2 F(u, 0) \int_{\alpha(\varepsilon, u)}^{1} \frac{\mathrm{d} v}{v^{2}} \mathrm{~d} u
\end{aligned}
$$

and where $F^{(1)}(u, 0)=F^{(2)}(u, 0)=F(u, 0)$. 
As typical in the direct approach, the first double integral $I_{0}$ is now regular for $\varepsilon \rightarrow 0$, while the other potentially singular integrals $I_{1}$ and $I_{2}$ are trivial functions of $v$ and can always be integrated analytically, yielding

$$
\int_{\alpha(\varepsilon, u)}^{1} \frac{\mathrm{d} v}{v}=-\ln |\alpha(\varepsilon, u)|=\ln |2 a(u)|-\ln |\varepsilon|+\mathrm{O}(\varepsilon)
$$

and

$$
\int_{\alpha(\varepsilon, u)}^{1} \frac{\mathrm{d} v}{v^{2}}=\frac{1}{\alpha(\varepsilon, u)}-1=\frac{2 a(u)}{\varepsilon}-\frac{u}{a(u)} \frac{\mathrm{d} a}{\mathrm{~d} u}(u)-1+\mathrm{O}(\varepsilon),
$$

where the final expressions have been obtained simply by inserting expansions (48).

For the treatment of $I_{0}$ and $I_{1}$ we can take advantage of expression (45) for the sum of the first derivatives of $F$. Hence we have for $I_{0}$

$$
\begin{aligned}
I_{0} & =\int_{0}^{1}\left\{\int_{-1}^{1}\left[F^{(1)}(u, v)+F^{(2)}(u, v)-2 F(u, 0)+2 \frac{\mathrm{d}}{\mathrm{d} u}(u F(u, 0)) v\right] \mathrm{d} u\right\} \frac{\mathrm{d} v}{v^{2}}+\mathrm{O}(\varepsilon) \\
& =\int_{0}^{1}\left\{\int_{-1}^{1}\left[F^{(1)}(u, v)+F^{(2)}(u, v)-2 F(u, 0)\right] \mathrm{d} u+2[F(1,0)+F(-1,0)] v\right\} \frac{\mathrm{d} v}{v^{2}}+\mathrm{O}(\varepsilon) .
\end{aligned}
$$

Similarly, for $I_{1}$ (using Eq. (51))

$$
\begin{aligned}
I_{1}= & -2 \int_{-1}^{1}\left\{\frac{\mathrm{d}}{\mathrm{d} u}(u F(u, 0)) \int_{\alpha(\varepsilon, u)}^{1} \frac{\mathrm{d} v}{v}\right\} \mathrm{d} u=2 \int_{-1}^{1}\left\{\frac{\mathrm{d}}{\mathrm{d} u}(u F(u, 0))\right\}[\ln |\varepsilon|-\ln |2 a(u)|] \mathrm{d} u+\mathrm{O}(\varepsilon) \\
= & 2\{\ln \varepsilon[F(1,0)+F(-1,0)]-F(1,0) \ln (2 a(1))-F(-1,0) \ln (2 a(-1))\} \\
& +2 \int_{-1}^{1}\left[u F(u, 0) \frac{1}{a(u)} \frac{\mathrm{d} a}{\mathrm{~d} u}(u)\right] \mathrm{d} u+\mathrm{O}(\varepsilon) .
\end{aligned}
$$

As we can see, the final expressions in (53) and (54) do not require the explicit knowledge of the derivatives of $F$, but just of $F$ itself. This confirms what was anticipated in the comments to Eq. (45).

For $I_{2}$ the treatment is even simpler

$$
I_{2}=2 \int_{-1}^{1} F(u, 0)\left\{\int_{\alpha(\varepsilon, u)}^{1} \frac{\mathrm{d} v}{v^{2}}\right\} \mathrm{d} u=2 \int_{-1}^{1} F(u, 0)\left[\frac{2 a(u)}{\varepsilon}-\frac{u}{a(u)} \frac{\mathrm{d} a}{\mathrm{~d} u}(u)-1\right] \mathrm{d} u+\mathrm{O}(\varepsilon) .
$$

Combining Eqs. (54) and (55) yields:

$$
\begin{aligned}
I_{1}+I_{2}= & \frac{1}{\varepsilon} \int_{-1}^{1} 4 F(u, 0) a(u) \mathrm{d} u+2 \ln \varepsilon[F(1,0)+F(-1,0)] \\
& -2 \int_{-1}^{1} F(u, 0) \mathrm{d} u-2 F(1,0) \ln (2 a(1))-2 F(-1,0) \ln (2 a(-1))+\mathrm{O}(\varepsilon) .
\end{aligned}
$$

It should be noted that in Eq. (56) there are an $\mathrm{O}(\ln \varepsilon)$ term and an $\mathrm{O}\left(\varepsilon^{-1}\right)$ term. Their cancellation will be discussed in Sections 5.3 and 6.2. 


\section{Double integration over consecutive elements}

Let $E$ and $E^{\prime}$ be two consecutive boundary elements, i.e. two elements having a common endpoint, with $\boldsymbol{y} \in E$ and $\boldsymbol{x} \in E^{\prime}$. The singularity occurs when $\boldsymbol{x}=\boldsymbol{y}$, which may happen either when $(\eta, \xi)=(-1,1)$ or when $(\eta, \xi)=(1,-1)$, depending on the relative position of $E$ and $E^{\prime}$. Of course, there is no singularity in the common endpoint if $\psi \varphi=0$. To address conveniently the two cases $(\eta, \xi)=(1,-1)$ (adjacent elements) and $(\eta, \xi)=(-1,1)$ (transposed adjacent elements), let $E^{\prime \prime}, E, E^{\prime}$ denote three consecutive elements (Fig. 3). We then consider the adjacent and transposed integrals $I_{\mathrm{A}}$ and $I_{\mathrm{T}}$ :

$$
\begin{aligned}
& I_{\mathrm{A}}=\int_{E} \int_{E^{\prime}(\boldsymbol{y}, \varepsilon)} V_{i j}(\boldsymbol{y}, \boldsymbol{x}) n_{i}(\boldsymbol{y}) n_{j}^{\prime}(\boldsymbol{x}) \psi(\boldsymbol{y}) \varphi^{\prime}(\boldsymbol{x}) \mathrm{d} s_{x} \mathrm{~d} s_{y}, \\
& I_{\mathrm{T}}=\int_{E} \int_{E^{\prime \prime}(\boldsymbol{y}, \varepsilon)} V_{i j}(\boldsymbol{y}, \boldsymbol{x}) n_{i}(\boldsymbol{y}) n_{j}^{\prime \prime}(\boldsymbol{x}) \psi(\boldsymbol{y}) \varphi^{\prime \prime}(\boldsymbol{x}) \mathrm{d} s_{x} \mathrm{~d} s_{y},
\end{aligned}
$$

where $E^{\prime}(\boldsymbol{y}, \varepsilon)$ and $E^{\prime \prime}(\boldsymbol{y}, \varepsilon)$ are defined according to (21), i.e.

$$
E^{\prime}(\boldsymbol{y}, \varepsilon)=\left\{\boldsymbol{x} \in E^{\prime}:|\boldsymbol{x}-\boldsymbol{y}| \geqslant \varepsilon, \quad \text { with } \quad \boldsymbol{y} \in E\right\}
$$

and similarly for $E^{\prime \prime}$. Scalar or vector functions defined on $E^{\prime}, E^{\prime \prime}$ will be tagged with the corresponding superscript, e.g. $\boldsymbol{a}^{\prime \prime}(\xi)$ for the natural tangent on $E^{\prime \prime}$.

The distance vector $\boldsymbol{r}$ is given by expression (28) and $(r=0) \Longleftrightarrow(\boldsymbol{x}=\boldsymbol{y}) \Longleftrightarrow(\xi \eta=-1)$. Therefore, in the parameter space we have to integrate over the image of $E \times E^{\prime}(\boldsymbol{y}, \varepsilon)$, which is the square $(\eta, \xi) \in$ $[-1,1] \times[-1,1]$ minus a small vanishing region around vertex $(\eta, \xi)=(1,-1)$ (or vertex $(-1,1)$ for $E \times$ $\left.E^{\prime \prime}(\boldsymbol{y}, \varepsilon)\right)$.

The double adjacent integration (57) and (58) will be treated by subdividing again the square $(\eta, \xi) \in$ $[-1,1] \times[-1,1]$ into two triangular regions, labeled (1) and (2) as shown in Fig. 2. Moreover, the mappings $(\eta, \xi) \leftrightarrow(u, v)$ defined in (31) and (32) can be used in this case as well.

\subsection{Singularity at $(\eta, \xi)=(1,-1)$ (adjacent elements)}

We first deal with the integral (57), that is, with the singularity occurring at the point $(\eta, \xi)=(1,-1)$ (Fig. 4):

$$
I_{\mathrm{A}}=I_{\mathrm{A}}^{(1)}+I_{\mathrm{A}}^{(2)} \text {. }
$$

In this case the integration over region (1) is nonsingular and we will consider in detail only the integral $I_{\mathrm{A}}^{(2)}$ over the triangular region (2).

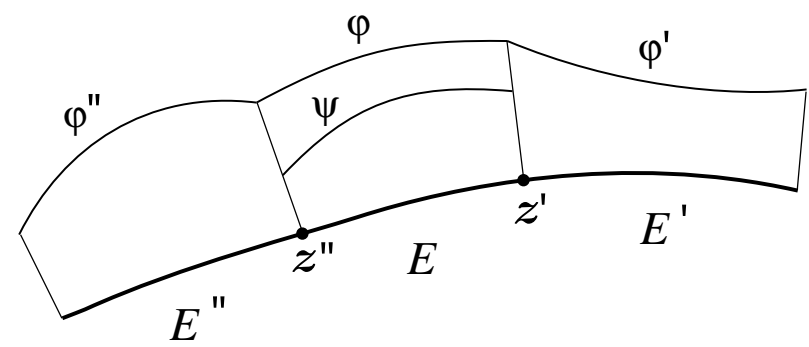

Fig. 3. Density and weight functions. 


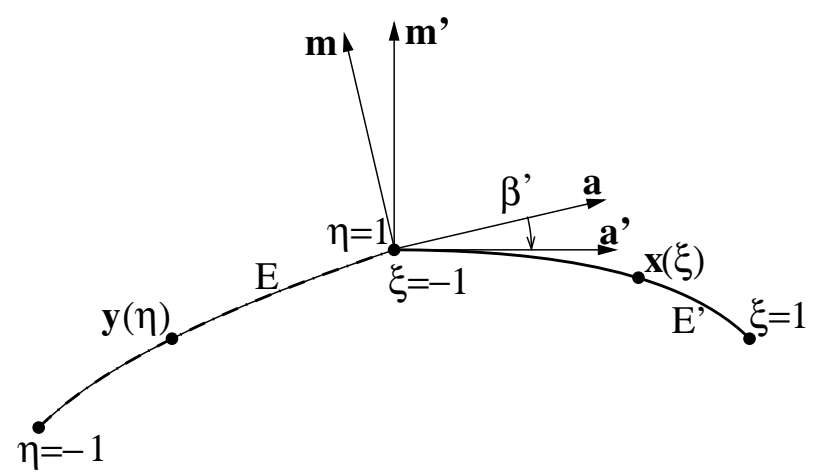

Fig. 4. Integration over adjacent elements $E$ and $E^{\prime}$.

If the mappings defined in (32) were directly employed, the singularity would occur at $v=1$. Therefore, it is merely a matter of convenience to shift and reverse the $v$ coordinate in (32) so that the singular point is defined by $v=0$ :

$$
\left\{\begin{array}{l}
\eta=(u-1) v+1=\eta_{\mathrm{A}}(u, v) \\
\xi=(u+1) v-1=\xi_{\mathrm{A}}(u, v)
\end{array} \quad \text { with } \quad-1 \leqslant u \leqslant 1,0 \leqslant v \leqslant 1\right.
$$

thus obtaining

$$
\mathrm{d} \xi \mathrm{d} \eta=2 v \mathrm{~d} u \mathrm{~d} v
$$

This coordinate transformation also lowers the order of singularity at $v=0$ (thus, e.g., regularizing completely the integral when a strongly singular kernel has to be integrated).

As in the coincident case, it is useful to introduce the Taylor expansion of $\boldsymbol{r}_{\mathrm{A}}(u, v)=\boldsymbol{x}\left(\xi_{\mathrm{A}}(u, v)\right)-$ $\boldsymbol{y}\left(\eta_{\mathrm{A}}(u, v)\right)$ as a function of $v$ and near $v=0$

$$
\begin{aligned}
\boldsymbol{r} & =\boldsymbol{r}_{\mathrm{A}}(u, v)=\boldsymbol{r}_{\mathrm{A}}(u, 0)+\left.\frac{\partial \boldsymbol{r}_{\mathrm{A}}}{\partial v}\right|_{v=0} v+\mathrm{O}\left(v^{2}\right)=0+\left[(u+1) \boldsymbol{a}^{\prime}-(u-1) \boldsymbol{a}\right] v+\mathrm{O}\left(v^{2}\right) \\
& =v\left[(u+1) \boldsymbol{a}^{\prime}-(u-1) \boldsymbol{a}+\mathrm{O}(v)\right]=v \hat{\boldsymbol{r}}_{\mathrm{A}}(u, v) .
\end{aligned}
$$

For brevity, $\boldsymbol{a}$ and $\boldsymbol{a}^{\prime}$ (without the argument $\xi$ or $\eta$ ) denote the natural tangents associated with $E$ and $E^{\prime}$ at their common endpoint $\boldsymbol{z}^{\prime}$, and similar notations will be used for other quantities as well (see Fig. 4).

Notice, importantly, that definition (62) of $\hat{\boldsymbol{r}}_{\mathrm{A}}(u, v)$ implies that $\hat{\boldsymbol{r}}_{\mathrm{A}}(u, 0)$ is never equal to zero. In fact, it is convenient to put $\hat{\boldsymbol{r}}_{\mathrm{A}}(u, 0)$ in the form:

$$
\hat{\boldsymbol{r}}_{\mathrm{A}}(u, 0)=s_{\mathrm{A}}(u) \boldsymbol{e}_{\mathrm{A}}(u),
$$

where $s_{\mathrm{A}}(u)$ is the norm of $\hat{\boldsymbol{r}}_{\mathrm{A}}(u, 0)$ :

$$
s_{\mathrm{A}}(u)=\left|\hat{\boldsymbol{r}}_{\mathrm{A}}(u, 0)\right|=a a^{\prime}\left[\chi^{\prime}(1+u)^{2}+2\left(1-u^{2}\right) \cos \beta^{\prime}+\chi^{\prime^{-1}}(1-u)^{2}\right]^{1 / 2}
$$

(having put $\beta^{\prime}=\left(\boldsymbol{a}, \boldsymbol{a}^{\prime}\right)$ and $\left.\chi^{\prime}=\left|\boldsymbol{a}^{\prime}\right| /|\boldsymbol{a}|=a^{\prime} / a\right)$ and $\boldsymbol{e}_{\mathrm{A}}(u)=\hat{\boldsymbol{r}}_{\mathrm{A}}(u, 0) / s_{\mathrm{A}}(u)$ is a unit vector. Moreover, putting $\hat{r}_{\mathrm{A}}(u, v)=\left|\hat{\boldsymbol{r}}_{\mathrm{A}}(u, v)\right|$, the distance $r=|\boldsymbol{r}|$ has the expansion

$$
r=v \hat{r}_{\mathrm{A}}(u, v)=v s_{\mathrm{A}}(u)+\mathrm{O}\left(v^{2}\right) .
$$


The integrand function in (57) as a function of $u$ and $v$ becomes

$$
\begin{aligned}
V_{i j}(\boldsymbol{y}, \boldsymbol{x}) n_{i}(\boldsymbol{y}) n_{j}^{\prime}(\boldsymbol{x}) \psi(\boldsymbol{y}) \varphi^{\prime}(\boldsymbol{x}) \mathrm{d} s_{x} \mathrm{~d} s_{y}= & \frac{1}{r^{2}} \mathscr{V}_{i j}(\boldsymbol{e}, r, \boldsymbol{y}(\eta)) m_{i}(\eta) m_{j}^{\prime}(\xi) \psi(\eta) \varphi^{\prime}(\xi) \mathrm{d} \xi \mathrm{d} \eta \\
= & \frac{1}{v^{2} \hat{r}_{\mathrm{A}}^{2}(u, v)} \mathscr{V}_{i j}\left(\boldsymbol{e}_{\mathrm{A}}(u, v), r_{\mathrm{A}}(u, v), \boldsymbol{y}\left(\eta_{\mathrm{A}}(u, v)\right)\right) m_{i}\left(\eta_{\mathrm{A}}(u, v)\right) \\
& \times m_{j}^{\prime}\left(\xi_{\mathrm{A}}(u, v)\right) \psi\left(\eta_{\mathrm{A}}(u, v)\right) \varphi^{\prime}\left(\xi_{\mathrm{A}}(u, v)\right) 2 v \mathrm{~d} u \mathrm{~d} v \\
= & \frac{1}{v} Q_{\mathrm{A}}(u, v) \mathrm{d} u \mathrm{~d} v
\end{aligned}
$$

which defines the nonsingular function $Q_{\mathrm{A}}(u, v)$. Here a first-order Taylor expansion of $Q_{\mathrm{A}}(u, v)$ around $v=0$ is sufficient

$$
Q_{\mathrm{A}}(u, v)=Q_{\mathrm{A}}(u, 0)+\mathrm{O}(v)
$$

where the term $Q_{\mathrm{A}}(u, 0)$ is easily found to be

$$
Q_{\mathrm{A}}(u, 0)=\frac{2}{s_{\mathrm{A}}^{2}(u)} \mathscr{V}_{i j}\left(\boldsymbol{e}_{\mathrm{A}}(u), 0, \boldsymbol{z}^{\prime}\right) m_{i}(-1) m_{j}^{\prime}(1) \psi(-1) \varphi^{\prime}(1) .
$$

It is worth noting that, whenever $\psi(1) \varphi^{\prime}(-1)=0$, we have $Q_{\mathrm{A}}(u, 0)=0$ and the integral is not singular.

The integration region $E \times E^{\prime}(\varepsilon, y)$ is characterized by the condition $r \geqslant \varepsilon$ which, together with expansion (65), becomes

$$
v \geqslant v_{\mathrm{A}}(u, \varepsilon) \quad \text { with } \quad v_{\mathrm{A}}(u, \varepsilon)=\frac{\varepsilon}{s_{\mathrm{A}}(u)}+\mathrm{O}\left(\varepsilon^{2}\right) .
$$

According to Eqs. (66) and (69), the integral (57) over adjacent elements becomes

$$
I_{\mathrm{A}}=I_{\mathrm{A}}^{(1)}+I_{\mathrm{A}}^{(2)}=I_{\mathrm{A}}^{(1)}+\int_{-1}^{1}\left\{\int_{\varepsilon / s_{\mathrm{A}}(u)}^{1} \frac{1}{v} Q_{\mathrm{A}}(u, v) \mathrm{d} v\right\} \mathrm{d} u+\mathrm{O}(\varepsilon) .
$$

Following the direct approach, the first term of expansion (67) is added and subtracted in (70), leading to:

$$
I_{\mathrm{A}}^{(2)}=\int_{-1}^{1} \int_{\varepsilon /\left(2 s_{\mathrm{A}}(u)\right)}^{1} \frac{1}{v}\left[Q_{\mathrm{A}}(u, v)-Q_{\mathrm{A}}(u, 0)\right] \mathrm{d} v \mathrm{~d} u+\int_{-1}^{1} Q_{\mathrm{A}}(u, 0) \mathrm{d} u \int_{\varepsilon / s_{\mathrm{A}}(u)}^{1} \frac{\mathrm{d} v}{v}+\mathrm{O}(\varepsilon),
$$

with $Q_{\mathrm{A}}(u, 0)$ given by (68). The first integral is nonsingular, while the second integral is singular but trivial in $v$ :

$$
\int_{\varepsilon / s_{\mathrm{A}}(u)}^{1} \frac{\mathrm{d} v}{v}=\ln \left|s_{\mathrm{A}}(u)\right|-\ln |\varepsilon| .
$$

This result, inserted in Eq. (71), provides the following expression for the adjacent double integral

$$
I_{\mathrm{A}}^{(2)}=\int_{-1}^{1} \int_{0}^{1} \frac{1}{v}\left[Q_{\mathrm{A}}(u, v)-Q_{\mathrm{A}}(u, 0)\right] \mathrm{d} v \mathrm{~d} u+\int_{-1}^{1} Q_{\mathrm{A}}(u, 0) \ln \left|s_{\mathrm{A}}(u)\right| \mathrm{d} u-\ln |\varepsilon| \int_{-1}^{1} Q_{\mathrm{A}}(u, 0) \mathrm{d} u+\mathrm{O}(\varepsilon) .
$$

\subsection{Singularity at $(\eta, \xi)=(1,-1)$ (transposed adjacent elements)}

We now deal with the integral (58), that is, with the singularity occurring at the point $(\eta, \xi)=(-1,1)$. In this case the integration over region (2) is nonsingular and only the integral $I_{\mathrm{T}}^{(1)}$ over region (1) is considered in some detail, following essentially the same steps as in Section 5.1. The coordinates defined in (31) are 
used, with translation and reversion of the $v$ coordinate in region (1) to have the singularity at $v=0$ (instead of $v=1)$ :

$$
\left\{\begin{array}{l}
\eta=(u+1) v-1=\eta_{\mathrm{T}}(u, v) \\
\xi=(u-1) v+1=\xi_{\mathrm{T}}(u, v)
\end{array} \quad \text { with } \quad u \in[-1,1] \quad \text { and } v \in[0,1]\right.
$$

thus obtaining

$$
\mathrm{d} \xi \mathrm{d} \eta=2 v \mathrm{~d} u \mathrm{~d} v
$$

These coordinate transformations are those in (60) with $\eta$ and $\xi$ switched:

$$
\eta_{\mathrm{T}}(u, v)=\xi_{\mathrm{A}}(u, v) \quad \xi_{\mathrm{T}}(u, v)=\eta_{\mathrm{A}}(u, v) .
$$

As before, the Taylor expansion of $\boldsymbol{r}_{\mathrm{T}}(u, v)=\boldsymbol{x}\left(\xi_{\mathrm{T}}(u, v)\right)-\boldsymbol{y}\left(\eta_{\mathrm{T}}(u, v)\right)$ as a function of $v$ and near $v=0$ is needed:

$$
\begin{aligned}
\boldsymbol{r} & =\boldsymbol{r}_{\mathrm{T}}(u, v)=\boldsymbol{r}_{\mathrm{T}}(u, 0)+\left.\frac{\partial \boldsymbol{r}_{\mathrm{T}}}{\partial v}\right|_{v=0} v+\mathrm{O}\left(v^{2}\right)=0-\left[-(u-1) \boldsymbol{a}^{\prime \prime}+(u+1) \boldsymbol{a}\right] v+\mathrm{O}\left(v^{2}\right) \\
& =-v\left[(1-u) \boldsymbol{a}^{\prime \prime}+(1+u) \boldsymbol{a}+\mathrm{O}(v)\right]=-v \hat{\boldsymbol{r}}_{\mathrm{T}}(u, v),
\end{aligned}
$$

where $\boldsymbol{a}^{\prime \prime}$ and $\boldsymbol{a}$ are the natural tangent vectors (23) to the elements $E^{\prime \prime}$ and $E$ at their shared endpoint $\boldsymbol{z}^{\prime \prime}$. Again, $\hat{\boldsymbol{r}}_{\mathrm{T}}(u, 0) \neq 0$ and it is convenient to put $\hat{\boldsymbol{r}}_{\mathrm{T}}(u, 0)$ in the form:

$$
\hat{\boldsymbol{r}}_{\mathrm{T}}(u, 0)=s_{\mathrm{T}}(u) \boldsymbol{e}_{\mathrm{T}}(u),
$$

where $s_{\mathrm{T}}(u)$ is the norm of $\hat{\boldsymbol{r}}_{\mathrm{T}}(u, 0)$ and $\boldsymbol{e}_{\mathrm{T}}(u)=\hat{\boldsymbol{r}}_{\mathrm{T}}(u, 0) / s_{\mathrm{T}}(u)$ is a unit vector. Moreover, the distance $r_{\mathrm{T}}=\left|\boldsymbol{r}_{\mathrm{T}}\right|$ has the expansion

$$
r_{\mathrm{T}}=v \hat{r}_{\mathrm{T}}(u, v)=v s_{\mathrm{T}}(u)+\mathrm{O}\left(v^{2}\right) \text {. }
$$

The integrand function in (58) as a function of $u$ and $v$ becomes

$$
\begin{aligned}
V_{i j}(\boldsymbol{y}, \boldsymbol{x}) n_{i}(\boldsymbol{y}) n_{j}^{\prime \prime}(\boldsymbol{x}) \psi(\boldsymbol{y}) \varphi^{\prime \prime}(\boldsymbol{x}) \mathrm{d} s_{x} \mathrm{~d} s_{y}= & \frac{1}{r^{2}} \mathscr{V}_{i j}(\boldsymbol{e}, r, \boldsymbol{y}(\eta)) m_{i}(\eta) m_{j}^{\prime \prime}(\xi) \psi(\eta) \varphi^{\prime \prime}(\xi) \mathrm{d} \xi \mathrm{d} \eta \\
= & \frac{1}{v^{2} \hat{r}_{\mathrm{T}}^{2}(u, v)} \mathscr{V}_{i j}\left(\boldsymbol{e}_{\mathrm{T}}(u, v), r_{\mathrm{T}}(u, v), \boldsymbol{y}\left(\eta_{\mathrm{T}}(u, v)\right)\right) m_{i}\left(\eta_{\mathrm{T}}(u, v)\right) \\
& \times m_{j}^{\prime \prime}\left(\xi_{\mathrm{T}}(u, v)\right) \psi\left(\eta_{\mathrm{T}}(u, v)\right) \varphi^{\prime \prime}\left(\xi_{\mathrm{T}}(u, v)\right) 2 v \mathrm{~d} u \mathrm{~d} v \\
= & \frac{1}{v} Q_{\mathrm{T}}(u, v) \mathrm{d} u \mathrm{~d} v .
\end{aligned}
$$

The integration region is again characterized by Eq. (69). Accordingly, the singular integral (58) can be rewritten in the following form

$$
I_{\mathrm{T}}=I_{\mathrm{T}}^{(1)}+I_{\mathrm{T}}^{(2)}=\int_{-1}^{1}\left\{\int_{\varepsilon / s_{\mathrm{T}}(u)}^{1} \frac{1}{v} Q_{\mathrm{T}}(u, v) \mathrm{d} v\right\} \mathrm{d} u+I_{\mathrm{T}}^{(2)}+\mathrm{O}(\varepsilon) .
$$

The first-order term in the Taylor expansion of $Q_{\mathrm{T}}(u, v)$ around $v=0$ is

$$
Q_{\mathrm{T}}(u, 0)=\frac{2}{s_{\mathrm{T}}^{2}(u)} \mathscr{V}_{i j}\left(\boldsymbol{e}_{\mathrm{T}}(u), 0, \boldsymbol{z}^{\prime \prime}\right) m_{i}(-1) m_{j}^{\prime \prime}(1) \psi(-1) \varphi^{\prime \prime}(1)
$$


Upon application of the direct method we obtain the following expression for the (transposed) adjacent double integral

$$
I_{\mathrm{T}}^{(1)}=\int_{-1}^{1}\left\{\int_{0}^{1} \frac{1}{v}\left[Q_{\mathrm{T}}(u, v)-Q_{\mathrm{T}}(u, 0)\right] \mathrm{d} v+Q_{\mathrm{T}}(u, 0) \ln \left|s_{\mathrm{T}}(u)\right|\right\} \mathrm{d} u-\ln |\varepsilon| \int_{-1}^{1} Q_{\mathrm{T}}(u, 0) \mathrm{d} u+\mathrm{O}(\varepsilon) .
$$

If $\psi \varphi_{\mathrm{T}}=0, Q_{\mathrm{T}}(u, 0)=0$ and the integral is not singular.

\subsection{Cancellation of $\ln |\varepsilon|$ terms}

From Eqs. (56), (72) and (81) we see that, for any given weight function $\psi$ on $E$, the coincident, adjacent and transposed-adjacent types of integration all give rise to $\ln |\varepsilon|$ terms, whose magnitude depend only on the element geometry and density values at element $E$ endpoints (Fig. 3). Adding all these contributions for the case of $\psi$ defined on $E$ yields

$$
\begin{aligned}
\ln |\varepsilon| \psi(1) & \left\{\mathscr{V}_{i j}\left(\boldsymbol{t}(1), 0, \boldsymbol{z}^{\prime}\right) n_{i}(1) n_{j}(1) \varphi(1)\right. \\
- & \left.\int_{-1}^{1} \frac{2}{s_{\mathrm{A}}^{2}(u)} \mathscr{V}_{i j}\left(\boldsymbol{e}_{\mathrm{A}}(u), 0, \boldsymbol{z}^{\prime}\right) m_{i}(1) m_{j}^{\prime}(-1) \varphi^{\prime}(-1) \mathrm{d} u\right\} \\
+ & \ln |\varepsilon| \psi(-1)\left\{\mathscr{V}_{i j}\left(\boldsymbol{t}(-1), 0, \boldsymbol{z}^{\prime \prime}\right) n_{i}(-1) n_{j}(-1) \varphi(-1)\right. \\
& \left.-\int_{-1}^{1} \frac{2}{s_{\mathrm{T}}^{2}(u)} \mathscr{V}_{i j}\left(\boldsymbol{e}_{\mathrm{T}}(u), 0, \boldsymbol{z}^{\prime \prime}\right) m_{i}(-1) m_{j}^{\prime \prime}(1) \varphi^{\prime \prime}(1) \mathrm{d} u\right\} .
\end{aligned}
$$

Each term within curly braces in Eq. (82) is zero provided the hypersingular kernels satisfy the following conditions (which constitute indeed some of their properties)

$$
\begin{aligned}
& \mathscr{V}_{i j}\left(\boldsymbol{t}(1), 0, \boldsymbol{z}^{\prime}\right) n_{i}(1) n_{j}(1)=\int_{-1}^{1} \frac{2}{s_{\mathrm{A}}^{2}(u)} \mathscr{V}_{i j}\left(\boldsymbol{e}_{\mathrm{A}}(u), 0, \boldsymbol{z}^{\prime}\right) m_{i}(1) m_{j}^{\prime}(-1) \mathrm{d} u, \\
& \mathscr{V}_{i j}\left(\boldsymbol{t}(-1), 0, \boldsymbol{z}^{\prime \prime}\right) n_{i}(-1) n_{j}(-1)=\int_{-1}^{1} \frac{2}{s_{\mathrm{T}}^{2}(u)} \mathscr{V}_{i j}\left(\boldsymbol{e}_{\mathrm{T}}(u), 0, \boldsymbol{z}^{\prime \prime}\right) m_{i}(-1) m_{j}^{\prime \prime}(1) \mathrm{d} u
\end{aligned}
$$

and in the algorithm the density function $\varphi$ is $C^{0}$ (continuous) across elements, i.e. $\varphi^{\prime \prime}(1)=\varphi(-1)$ and $\varphi(1)=\varphi^{\prime}(-1)$. It is worth remarking that the $C^{1, \alpha}$ smoothness required at $\varphi$ at $\boldsymbol{y}$ (cf. Eq. (15)) has no relevance here since $\boldsymbol{y}$ is never an endpoint for $E$. Therefore, $\varphi$ must be $C^{1, \alpha}$ strictly inside each boundary element (where it is usually $C^{\infty}$ ) and $C^{0}$ at both endpoints.

For collocation BEM, an in-depth discussion of these cancellations conditions can be found in [30].

\section{Evaluation of free terms due to the hypersingularity}

Let us go back to the starting identity (18) for the Galerkin BEM. The following free term

$$
\int_{\Gamma} \psi(\boldsymbol{y}) \varphi(\boldsymbol{y})\left\{\int_{s_{\varepsilon}} V(\boldsymbol{y}, \boldsymbol{x}) \mathrm{d} s_{x}\right\} \mathrm{d} s_{y}
$$

appeared in Eq. (18) and needs further treatment to obtain its explicit expression. Once the boundary $\Gamma$ has been subdivided into boundary elements, one is led to compute the limiting form of the element-wise versions of (85) 


$$
H=\int_{E} \psi(\boldsymbol{y}) \varphi(\boldsymbol{y})\left\{\int_{s_{\varepsilon}} V(\boldsymbol{y}, \boldsymbol{x}) \mathrm{d} s_{x}\right\} \mathrm{d} s_{y} .
$$

Note that both $\psi$ and $\varphi$ are defined on $E$.

It will prove useful to describe points $\boldsymbol{x} \in s_{\varepsilon}$ by their polar coordinates $(\varepsilon, \omega)$ relative to the orthonormal frame $\boldsymbol{t}(\eta), \boldsymbol{n}(\eta)$ :

$$
\boldsymbol{x}-\boldsymbol{y}(\eta)=\varepsilon \boldsymbol{e}(\omega ; \eta), \quad \boldsymbol{e}(\omega ; \eta)=\boldsymbol{t}(\eta) \cos \omega+\boldsymbol{n}(\eta) \sin \omega,
$$

so that

$$
\mathrm{d} s_{x}=\varepsilon \mathrm{d} \omega, \quad \boldsymbol{n}(\boldsymbol{x})=-\boldsymbol{e}(\omega ; \eta) .
$$

In particular, let $\omega=\theta^{ \pm}(\eta, \varepsilon)$ denote the polar angles defining the two intersection points $\boldsymbol{x}^{ \pm}(\eta, \varepsilon)$ of $s_{\varepsilon}$ with $\Gamma$ (Fig. 5), i.e.:

$$
\boldsymbol{x}^{ \pm}(\eta, \varepsilon)=\boldsymbol{y}(\eta)+\varepsilon \boldsymbol{e}\left(\theta^{ \pm}(\eta, \varepsilon) ; \eta\right) .
$$

Introducing (87) and the representation (3) and (7) of the kernel and taking (88) into account, the free term (86) becomes:

$$
H=\frac{1}{\varepsilon} \int_{-1}^{1} \psi(\eta) \varphi(\eta) m_{i}(\eta)\left[\mathscr{H}_{i}\left(\theta^{+}, \varepsilon, \eta\right)-\mathscr{H}_{i}\left(\theta^{-}, \varepsilon, \eta\right)\right] \mathrm{d} \eta
$$

having put

$$
\mathscr{H}_{i}(\omega, \varepsilon, \eta)=-\int_{0}^{\omega} \mathscr{V}_{i j}\left(\boldsymbol{e}\left(\omega^{\prime} ; \eta\right), \varepsilon, \boldsymbol{y}(\eta)\right) e_{j}\left(\omega^{\prime} ; \eta\right) \mathrm{d} \omega^{\prime} .
$$

In this section, we use notations $E^{-}, E, E^{+}$instead of $E^{\prime \prime}, E, E^{\prime}$ and corresponding superscripts for quantities associated to an element, both to emphasize that we are here integrating over the single element $E$ instead of over a product of elements and to deal at once with both endpoint contributions.

For a fixed $\varepsilon>0$, the point $\boldsymbol{x}^{+}(\eta, \varepsilon)$ belongs to either $E$ or $E^{+}$, depending on how close $\boldsymbol{y}$ is to the endpoint $\boldsymbol{z}^{+}$of $E$ (Figs. 5 and 7); likewise, $\boldsymbol{x}^{-}(\eta, \varepsilon)$ belongs to either $E$ or $E^{-}$. It is therefore convenient to put (Fig. 6)

$$
D_{\varepsilon}^{ \pm}=\left\{-1 \leqslant \eta \leqslant 1:\left|\boldsymbol{y}(\eta)-\boldsymbol{z}^{ \pm}\right| \leqslant \varepsilon\right\}, \quad E_{\varepsilon}^{ \pm}=[-1,1] \backslash D_{\varepsilon}^{ \pm},
$$
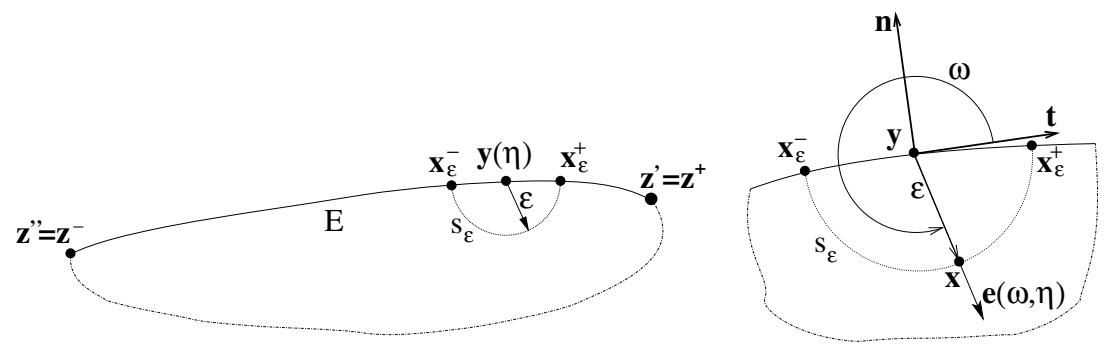

Fig. 5. Free term evaluation: coincident case.

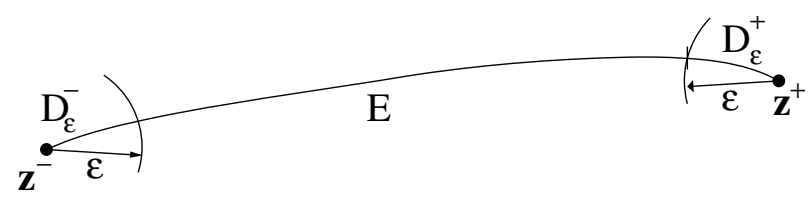

Fig. 6. Definition of $D_{\varepsilon}^{-}$and $D_{\varepsilon}^{+}$. 


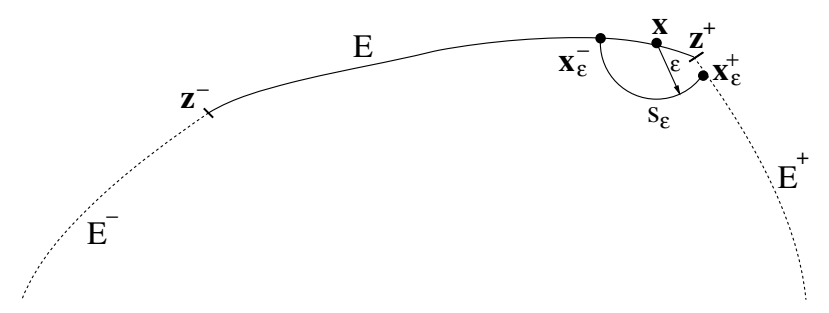

Fig. 7. Free term evaluation: adjacent case.

and split the integral (89) into contributions of coincident type $H_{\mathrm{c}}^{ \pm}(\varepsilon)$ and of adjacent type $H_{\mathrm{a}}^{ \pm}(\varepsilon)$ :

$$
H(\varepsilon)=H_{\mathrm{c}}^{+}(\varepsilon)-H_{\mathrm{c}}^{-}(\varepsilon)+H_{\mathrm{a}}^{+}(\varepsilon)-H_{\mathrm{a}}^{-}(\varepsilon)
$$

with

$$
\begin{aligned}
& H_{\mathrm{c}}^{ \pm}(\varepsilon)=\frac{1}{\varepsilon} \int_{E_{\varepsilon}^{ \pm}} \psi(\eta) \varphi(\eta) m_{i}(\eta) \mathscr{H}_{i}\left(\theta^{ \pm}(\eta, \varepsilon), \varepsilon, \eta\right) \mathrm{d} \eta, \\
& H_{\mathrm{a}}^{ \pm}(\varepsilon)=\frac{1}{\varepsilon} \int_{D_{\varepsilon}^{ \pm}} \psi(\eta) \varphi(\eta) m_{i}(\eta) \mathscr{H}_{i}\left(\theta^{ \pm}(\eta, \varepsilon), \varepsilon, \eta\right) \mathrm{d} \eta .
\end{aligned}
$$

The integrals in (92) and (93) depend on $\varepsilon$. However, on using the coordinates $(u, v)$ introduced in Sections 4 and $5, H_{\mathrm{c}}^{ \pm}(\varepsilon)$ and $H_{\mathrm{a}}^{ \pm}(\varepsilon)$, respectively, can be reformulated as integrals over a fixed interval $u \in[-1,1]$. This treatment, which provides a convenient way to evaluate the hypersingular free term, is now going to be presented in some detail.

\subsection{Contributions of coincident type}

The contributions of coincident type, namely $H_{\mathrm{c}}^{ \pm}(\varepsilon)$, are expected to be of order $\mathrm{O}(1 / \varepsilon)$ for vanishingly small $\varepsilon$. Thus, two-term expansions about $\varepsilon=0$ are needed in order to evaluate both the unbounded $\mathrm{O}(1 / \varepsilon)$ and the bounded $\mathrm{O}(1)$ contributions to $H_{\mathrm{c}}^{ \pm}(\varepsilon)$.

The integral $H_{\mathrm{c}}^{ \pm}(\varepsilon)$ defined by (92) involves $\boldsymbol{y}(\eta)$ and, through the angle $\theta^{ \pm}, \boldsymbol{x}_{\varepsilon}^{ \pm}(\boldsymbol{y}(\eta))$. The coordinates $(u, v)$ defined in Section 4 for the coincident double integration are again used. In particular, the position vector $\boldsymbol{r}^{ \pm}=\boldsymbol{x}^{ \pm}(\eta, \varepsilon)-\boldsymbol{y}(\eta)$ is still expressed in terms of $(u, v)$ by (37), where, in addition, $v, \varepsilon$ and $u$ are linked by (48) in order to satisfy the condition $\left|\boldsymbol{r}^{ \pm}\right|=\varepsilon$ (where the \pm sign corresponds to that in $H_{\mathrm{c}}^{ \pm}(\varepsilon)$ ). Eliminating $v$ in (37) by using (48) and taking the Taylor expansion about $\varepsilon=0$ of the resulting expression of $\boldsymbol{r}^{ \pm}$yields:

$$
\begin{aligned}
\boldsymbol{r}^{ \pm} & = \pm \varepsilon\left[\boldsymbol{t}(u)+\left(\frac{1}{2 a^{2}(u)} \frac{\mathrm{d} a}{\mathrm{~d} u}(u) \boldsymbol{t}(u)-\frac{1}{2 a^{2}(u)} \boldsymbol{b}(u)\right) u \varepsilon+\mathrm{O}\left(\varepsilon^{2}\right)\right] \\
& = \pm \varepsilon\left[\boldsymbol{t}(u)-\frac{u}{2 a^{2}(u)}(\boldsymbol{b}(u) \cdot \boldsymbol{n}(u)) \boldsymbol{n}(u) \varepsilon+\mathrm{O}\left(\varepsilon^{2}\right)\right] .
\end{aligned}
$$

The coordinate $\eta$ itself becomes, by virtue of (31) and (48), a function of $u$ and $\varepsilon$ :

$$
\eta=\eta^{ \pm}(u, v(u, \varepsilon))=u+( \pm 1-u) v(u, \varepsilon)=u+\frac{( \pm 1-u)}{2 a(u)} \varepsilon+\mathrm{O}\left(\varepsilon^{2}\right) \equiv u+\delta^{ \pm}(u) \varepsilon+\mathrm{O}\left(\varepsilon^{2}\right),
$$

while the differential $\mathrm{d} \eta$ is given by: 


$$
\mathrm{d} \eta=\frac{\partial \eta^{ \pm}}{\partial u} \mathrm{~d} u=\left[1+\frac{\mathrm{d}}{\mathrm{d} u} \delta^{ \pm}(u) \varepsilon\right] \mathrm{d} u+\mathrm{O}\left(\varepsilon^{2}\right)
$$

On the other hand, from (87) and the definition of $\theta^{ \pm}$, one must have:

$$
\boldsymbol{r}^{ \pm}=\varepsilon \boldsymbol{e}\left(\theta^{ \pm} ; \eta\right) .
$$

Expressions (94) and (97) are found to coincide if $\theta^{+}$and $\theta^{-}$are given by:

$$
\theta^{+}(u, \varepsilon)=-\kappa(u) \varepsilon+\mathrm{O}\left(\varepsilon^{2}\right), \quad \theta^{-}(u, \varepsilon)=-\pi+\kappa(u) \varepsilon+\mathrm{O}\left(\varepsilon^{2}\right)
$$

with

$$
\kappa(u)=-\frac{u}{2 a^{2}(u)}(\boldsymbol{b}(u) \cdot \boldsymbol{n}(u)) .
$$

Using $u$, spanning the fixed interval $[-1,1]$, as the integration variable, the integral $H_{\mathrm{c}}^{ \pm}(\varepsilon)$ takes the form:

$$
H_{\mathrm{c}}^{ \pm}(\varepsilon)=\frac{1}{\varepsilon} \int_{-1}^{1}\left[\psi \varphi m_{i}\right]\left(\eta^{ \pm}(u, \varepsilon)\right) \mathscr{H}_{i}\left(\theta^{ \pm}(u, \varepsilon), \varepsilon, \eta^{ \pm}(u, \varepsilon)\right) \frac{\partial \eta^{ \pm}}{\partial u} \mathrm{~d} u
$$

which facilitates the investigation of its behaviour about $\varepsilon=0$. Indeed, the factor of $1 / \varepsilon$ in the expression (99) of $H_{\mathrm{c}}^{ \pm}(\varepsilon)$ is bounded and has a bounded derivative w.r.t. $\varepsilon$ at $\varepsilon=0$. Thus, $H_{\mathrm{c}}^{ \pm}(\varepsilon)$ admits an expansion of the form:

$$
\begin{aligned}
H_{\mathrm{c}}^{ \pm}(\varepsilon)= & \frac{1}{\varepsilon} \int_{-1}^{1}\left\{\left[\psi \varphi m_{i}\right]\left(\eta^{ \pm}(u, 0)\right) \mathscr{H}_{i}\left(\theta^{ \pm}(u, 0), 0, \eta^{ \pm}(u, 0)\right) \frac{\partial \eta^{ \pm}}{\partial u}(u, 0)\right\} \mathrm{d} u \\
& +\left.\int_{-1}^{1} \frac{\partial}{\partial \varepsilon}\left\{\left[\psi \varphi m_{i}\right]\left(\eta^{ \pm}(u, \varepsilon)\right) \mathscr{H}_{i}\left(\theta^{ \pm}(u, \varepsilon), \varepsilon, \eta^{ \pm}(u, \varepsilon)\right) \frac{\partial \eta^{ \pm}}{\partial u}\right\}\right|_{\varepsilon=0} \mathrm{~d} u+\mathrm{O}(\varepsilon) .
\end{aligned}
$$

For $\varepsilon=0$, one has $\eta^{ \pm}=u, \partial \eta^{ \pm} / \partial u=1, \theta^{+}=0, \theta^{-}=-\pi$. Hence:

$$
\left[\psi \varphi m_{i}\right]\left(\eta^{ \pm}(u, 0)\right) \mathscr{H}_{i}\left(\theta^{ \pm}(u, 0), 0, \eta^{ \pm}(u, 0)\right) \frac{\partial \eta^{ \pm}}{\partial u}(u, 0)=\left[\psi \varphi m_{i}\right](u) \mathscr{H}_{i}\left(\theta^{ \pm}, 0, u\right) .
$$

Moreover, for $\varepsilon=0$, one also has

$$
\left.\frac{\partial}{\partial \varepsilon} \mathscr{H}_{i}\left(\theta^{ \pm}(u, \varepsilon), \varepsilon, \eta^{ \pm}(u, \varepsilon)\right)\right|_{\varepsilon=0}=0
$$

by virtue of (12), and

$$
\frac{\partial}{\partial \varepsilon} \frac{\partial \eta^{ \pm}}{\partial u}=\frac{\mathrm{d}}{\mathrm{d} u} \delta^{ \pm}(u), \quad \frac{\partial \theta^{ \pm}}{\partial \varepsilon}=\mp \kappa(u), \quad \boldsymbol{e}\left(\theta^{ \pm}, \eta\right)= \pm \boldsymbol{t}(u), \quad \frac{\partial \mathscr{H}_{i}}{\partial \omega}\left(\theta^{ \pm}, 0, u\right)=\mp \mathscr{V}_{i j}( \pm \boldsymbol{t}(u), 0, \boldsymbol{y}(u)) t_{j}(u),
$$

where $\delta^{ \pm}(u)$ and $\kappa(u)$ are defined by (95) and (98), respectively; the last equality results directly from the definition (90) of $\mathscr{H}$. Using these equalities, one has:

$$
\begin{aligned}
\frac{\partial}{\partial \varepsilon}\{ & {\left.\left[\psi \varphi m_{i}\right]\left(\eta^{ \pm}(u, \varepsilon)\right) \mathscr{H}_{i}\left(\theta^{ \pm}(u, \varepsilon), \varepsilon, \eta^{ \pm}(u, \varepsilon)\right) \frac{\partial \eta^{ \pm}}{\partial u}\right\}\left.\right|_{\varepsilon=0} } \\
= & {\left[\psi \varphi m_{i}\right](u) \frac{\partial \theta^{ \pm}}{\partial \varepsilon}(u, \varepsilon) \frac{\partial \mathscr{H}_{i}}{\partial \omega}\left(\theta^{ \pm}, 0, u\right)+\left.\frac{\partial}{\partial \eta}\left\{\left[\psi \varphi m_{i}\right](\eta) \mathscr{H}_{i}\left(\theta^{ \pm}, 0, \eta\right)\right\}\right|_{\eta=u} \delta^{ \pm}(u) } \\
& +\left[\psi \varphi m_{i}\right](u) \mathscr{H}_{i}\left(\theta^{ \pm}, 0, u\right) \frac{\mathrm{d}}{\mathrm{d} u} \delta^{ \pm}(u) \\
= & -\left[\psi \varphi \kappa m_{i} t_{j}\right](u) \mathscr{V}_{i j}( \pm \boldsymbol{t}(u), 0, \boldsymbol{y}(u))+\frac{\partial}{\partial u}\left\{\left[\psi \varphi \delta^{ \pm} m_{i}\right](u) \mathscr{H}_{i}\left(\theta^{ \pm}, 0, u\right)\right\}
\end{aligned}
$$


Substituting Eqs. (101) and (102) into (100), using $\delta^{+}(1)=\delta^{-}(-1)=0$ and $\delta^{+}(-1)=1 / a^{-}, \delta^{-}(1)=$ $-1 / a^{+}$, one then obtains the desired expansion for the coincident contribution to the free term:

$$
\begin{aligned}
H_{\mathrm{c}}^{+}(\varepsilon)-H_{\mathrm{c}}^{-}(\varepsilon)= & \frac{1}{\varepsilon} \int_{-1}^{1}\left[\psi \varphi m_{i}\right](u)\left[\mathscr{H}_{i}(0,0, u)-\mathscr{H}_{i}(-\pi, 0, u)\right] \mathrm{d} u \\
& +\left[\psi \varphi n_{i}\right](1) \mathscr{H}_{i}(-\pi, 0,1)-\left[\psi \varphi n_{i}\right](-1) \mathscr{H}_{i}(0,0,-1)+\hat{H}+\mathrm{O}(\varepsilon)
\end{aligned}
$$

with

$$
\hat{H}=-\int_{-1}^{1}\left[\psi \varphi \kappa m_{i} t_{j}\right](u)\left[\mathscr{V}_{i j}(\boldsymbol{t}(u), 0, \boldsymbol{y}(u))-\mathscr{V}_{i j}(-\boldsymbol{t}(u), 0, \boldsymbol{y}(u))\right] \mathrm{d} u .
$$

The first line in this formula is the weighted form of the unbounded free term which occurs in the direct approach for the collocation hypersingular BEM [23]. The finite term appearing in the last line, on the other hand, is specific to the Galerkin formulation and cannot arise in (and be expected from the knowledge of) the collocation hypersingular BEM. Besides, Eq. (98) relies on the curvature being continuous inside the element $E$, so that the additional free term occurring in the collocation hypersingular BEM [25] does not arise here. The term $\hat{H}$ in practice vanishes except in quite special situations, e.g. a boundary element on an interface.

\subsection{Cancellation of $1 / \varepsilon$ terms}

The $\mathrm{O}\left(\varepsilon^{-1}\right)$ term in Eq. (103) must cancel exactly the corresponding unbounded term in Eq. (56), which comes from the double integration for the coincident case, for any choice of the density and weight functions. The fundamental solution is therefore required to verify (see also Eq. (44)):

$$
2 \mathscr{V}_{i j}(\boldsymbol{t}(u), 0, \boldsymbol{y}(u)) n_{i}(u) n_{j}(u)+\mathscr{H}_{i}(0,0, u) n_{i}(u)-\mathscr{H}_{i}(-\pi, 0, u) n_{i}(u)=0(-1 \leqslant u \leqslant 1) .
$$

It is worth noting that this cancellation does not put any interelement continuity requirement on $\varphi$.

\subsection{Contributions of adjacent type}

These contributions $H_{\mathrm{a}}^{ \pm}(\varepsilon)$, defined by (93), are associated with the right and left endpoints of $E$, as the + or - superscript (respectively) indicates. Again we found convenient to introduce the coordinates $(u, v)$ defined in Sections 5.1 and 5.2 for the double integration over adjacent elements. Thus, we have

$$
\eta=\eta^{ \pm}(u, v)=(u \mp 1) v \pm 1
$$

together with, again, the condition $\left|\boldsymbol{r}^{ \pm}=\varepsilon\right|$ which, from (60) and (73), makes $\eta$ to depend on $\varepsilon$ and $u$, so as to obtain:

$$
\eta=\eta^{ \pm}(\varepsilon, u)= \pm 1+(u \mp 1) \frac{\varepsilon}{s^{ \pm}(u)}+\mathrm{O}\left(\varepsilon^{2}\right) .
$$

Similarly, one obtains

$$
\begin{aligned}
\boldsymbol{r}^{ \pm}(\varepsilon, u) & =\frac{\varepsilon}{s^{ \pm}(u)}\left[(u \pm 1) \boldsymbol{a}^{ \pm}+( \pm 1-u) \boldsymbol{a}\right]+\mathrm{O}\left(\varepsilon^{2}\right) \\
& =\varepsilon \boldsymbol{e}\left(\theta^{ \pm}(u)\right)+\mathrm{O}\left(\varepsilon^{2}\right),
\end{aligned}
$$

where $\boldsymbol{a}$ and $\boldsymbol{a}^{ \pm}$are the natural tangent vectors of $E$ and the neighbouring elements $E^{ \pm}$at the junction point $\boldsymbol{z}^{ \pm}$between $E$ and $E^{ \pm}$, and

$$
s^{ \pm}(u)=\left|(u \pm 1) \boldsymbol{a}^{ \pm}+( \pm 1-u) \boldsymbol{a}\right| .
$$


Eq. (107) defines the functions $\theta^{ \pm}(u)$, which are such that:

$$
\theta^{ \pm}(\eta, \varepsilon)=\theta^{ \pm}(u)+\mathrm{O}(\varepsilon)
$$

If $\beta^{ \pm}=0$, i.e. if the unit tangent is continuous between elements $E$ and $E^{ \pm}$, the functions $\theta^{ \pm}(u)$ are constant: $\theta^{+}(u)=0$ and $\theta^{-}(u)=\pi$. Otherwise, using (107) and (108), one can also express $u$ and $s^{ \pm}(u)$ in terms of $\theta^{ \pm}$ through:

$$
\begin{aligned}
& u=u^{ \pm}\left(\theta^{ \pm}\right)= \pm \frac{a \sin \theta^{ \pm}+a^{ \pm} \sin \left(\theta^{ \pm} \mp \beta^{ \pm}\right)}{a \sin \theta^{ \pm}-a^{ \pm} \sin \left(\theta^{ \pm} \mp \beta^{ \pm}\right)}, \\
& s^{ \pm}(u)=\tilde{s}^{ \pm}\left(\theta^{ \pm}\right)=\frac{2 a a^{ \pm} \sin \beta^{ \pm}}{a \sin \theta^{ \pm}-a^{ \pm} \sin \left(\theta^{ \pm} \mp \beta^{ \pm}\right)} .
\end{aligned}
$$

For $u$ to span the interval $[-1,1]$, one has $\theta^{ \pm} \in\left[\theta_{1}^{ \pm}, \theta_{2}^{ \pm}\right]$, with $\theta_{1}^{+}=0, \theta_{2}^{+}=\beta^{+}, \theta_{1}^{-}=\pi-\beta^{-}, \theta_{2}^{-}=\pi$.

Now, the integration for $H_{\mathrm{a}}^{ \pm}(\varepsilon)$ can be carried out with respect to $u$ and with $u \in[-1,1]$, that is, with $u$ spanning between fixed values. The differential $\mathrm{d} \eta$ has the following expansion in $\varepsilon$ :

$$
\mathrm{d} \eta=\frac{\partial \eta^{ \pm}}{\partial u} \mathrm{~d} u=\varepsilon \frac{\mathrm{d}}{\mathrm{d} u}\left(\frac{u \mp 1}{s^{ \pm}(u)}\right) \mathrm{d} u+\mathrm{O}\left(\varepsilon^{2}\right) .
$$

Since this differential is $\mathrm{O}(\varepsilon)$, only the leading term of the expansion of the integrand function in (93) is sought. Thus:

$$
H_{\mathrm{a}}^{ \pm}(\varepsilon)=\left[\psi \varphi m_{i}\right]( \pm 1) \int_{-1}^{1} \mathscr{H}_{i}\left(\theta^{ \pm}(u), 0, \pm 1\right) \mathrm{d} \eta^{ \pm}(u)+\mathrm{O}(\varepsilon) .
$$

If $\beta^{ \pm}=0$, a simple calculation of the integral (113) using (112), $\theta^{+}(u)=0, \theta^{-}(u)=-\pi$ and $s^{+}(-1)=$ $s^{-}(1)=2 a$ yields the result:

$$
H_{\mathrm{a}}^{ \pm}(\varepsilon)=\mathscr{H}_{i}\left(\theta^{ \pm}, 0, \pm 1\right)\left[\psi \varphi n_{i}\right]( \pm 1)+\mathrm{O}(\varepsilon) .
$$

If $\beta^{ \pm} \neq 0$, it is natural to introduce the change of variables $u=u(\theta)$ as defined by (110) in (113) (note that $\theta$ also span fixed intervals). In particular, applying (110) and (111) to (112), one finds that:

$$
\mathrm{d} \eta^{ \pm}= \pm \frac{\cos \left(\theta \mp \beta^{ \pm}\right)}{a \sin \beta^{ \pm}} \mathrm{d} \theta
$$

and (93) becomes:

$$
H_{\mathrm{a}}^{ \pm}(\varepsilon)= \pm\left[\psi \varphi n_{i}\right]( \pm 1) \int_{\theta_{1}^{ \pm}}^{\theta_{2}^{ \pm}} \mathscr{H}_{i}(\theta, 0, \pm 1) \frac{\cos \left(\theta \mp \beta^{ \pm}\right)}{\sin \beta^{ \pm}} \mathrm{d} \theta+\mathrm{O}(\varepsilon) .
$$

Although this formula is, strictly speaking, valid only if $\beta^{ \pm} \neq 0$, its limiting value for $\beta^{ \pm}=0$ is easily shown via a Taylor expansion to be indeed (114).

\subsection{Hypersingular free term final expression}

The final formula for the element-wise free term (86) is obtained by substituting (103) and (116), in the limit $\varepsilon \rightarrow 0$ and dropping the $\mathrm{O}(1 / \varepsilon)$ term, into (91):

$$
H=H^{+}+H^{-}+\hat{H},
$$


where $\hat{H}$ is defined by (104) and with

$$
\begin{aligned}
& H^{+}=\left\{\mathscr{H}_{i}(-\pi, 0,1)+\int_{0}^{\beta^{\prime}} \mathscr{H}_{i}(\theta, 0,1) \frac{\cos \left(\theta-\beta^{\prime}\right)}{\sin \beta^{\prime}} \mathrm{d} \theta\right\}\left[\psi \varphi n_{i}\right](1), \\
& H^{-}=-\left\{\mathscr{H}_{i}(0,0,-1)+\int_{0}^{\beta^{\prime \prime}} \mathscr{H}_{i}(\pi-\theta, 0,-1) \frac{\cos \left(\theta-\beta^{\prime \prime}\right)}{\sin \beta^{\prime \prime}} \mathrm{d} \theta\right\}\left[\psi \varphi n_{i}\right](-1),
\end{aligned}
$$

using again the notations $\beta^{\prime}, \beta^{\prime \prime}$ instead of $\beta^{+}, \beta^{-}$. The change of variable $\theta \rightarrow \pi-\theta$ has been used in $H_{\mathrm{a}}^{-}$ defined by (116). The finite free term (117) is specific to Galerkin BEM and apparently had never been detected before.

The free terms $H^{+}, H^{-}$are of coincident type in that they involve only $\varphi$ and $\psi$ on $E$, but also of adjacent type in that they depend on the angles $\beta^{\prime}, \beta^{\prime \prime}$ with neighbouring elements. It is useful to note that, as a consequence of (114), $H^{+}+H^{-}=0$ if the tangent has interelement continuity. Since $\varphi$ must have interelement continuity (see Section 5.3), it is in practice more natural to treat $H^{+}, H^{-}$as adjacent terms. If we think of $\varphi$ as a continuous shape function, we see that it ought to be either $\varphi(-1)=0$ or $\varphi(1)=0$, which simplify the expression for the free term $H$. Moreover, if the support of a continuous weight function $\psi$ spans two boundary elements, we see that each side contributes a free term.

A short comment on the finite free terms just obtained is in order as they may look a bit unusual. They have never been detected before because in no other paper a limit process based on the vanishing neighbourhood approach has been used in conjunction with SGBEM. All formulations of SGBEM based on regularization of the kernels before performing the limit, like in [4-9], do not provide free terms at all (and this is indeed one of their advantages). The same is true for the limit to the boundary method [10-12]. On the other hand, techniques based on the finite part idea [13-15] completely overlook the free term evaluation, as the 'bump' associated to the vanishing neighbourhood is never taken into account.

The direct approach for the evaluation of singular integrals, here pursued and extended to SGBEM, is strongly based on a careful analysis of the limiting process, as the neighbourhood and the 'bump' around the singularity vanish, and free terms are therefore an essential part. It should also be considered that in the present paper the double integrations have been always dealt with as a whole, thus fully exploiting the features of the Galerkin BEM, and this aspect also affects the nature and the value of the free terms.

\subsection{Evaluation of the free term $\boldsymbol{c}(\boldsymbol{y})$}

Using the formalism introduced in this section, the free term $\boldsymbol{c}(\boldsymbol{y})$ defined by (17) becomes:

$$
\boldsymbol{c}(\boldsymbol{y})=\int_{-\pi}^{0}\{\mathscr{V}(\boldsymbol{e}(\omega ; \eta), 0, \boldsymbol{y}) \boldsymbol{e}(\omega ; \eta)-\mathscr{W}(\boldsymbol{e}(\omega ; \eta), 0, \boldsymbol{y}) \boldsymbol{n}(\boldsymbol{x})\} \mathrm{d} \omega
$$

where $\mathscr{W}(\boldsymbol{e}, r, \boldsymbol{y})=r W(\boldsymbol{y}, \boldsymbol{x})$ is the singular part of the strongly singular kernel $W(\boldsymbol{y}, \boldsymbol{x})$. Note that the integration bounds mean that $\Gamma$ is smooth at $y$. This is the only case needed in connection with the weighted identity (18) as long as the irregular points on $\Gamma$ are isolated (e.g. a finite number of corners). Since $\boldsymbol{c}(\boldsymbol{y})$ are regular functions, the evaluation of the integral

$$
\int_{\Gamma} \psi(\boldsymbol{y}) \boldsymbol{\nabla} \varphi(\boldsymbol{y}) \cdot \boldsymbol{c}(\boldsymbol{y}) \mathrm{d} s_{y}
$$

in the identity (18) is a trivial task. 


\section{The direct algorithm}

Summing up the analysis of Sections 4 and 6, the final formula for the coincident double integration over the element $E$ stems from Eqs. (50), (53), (56) and (117) and reads:

$$
I_{C}=\int_{0}^{1}\left\{\int_{-1}^{1}\left[F^{(1)}(u, v)+F^{(2)}(u, v)-2 F(u, 0)\right] \mathrm{d} u+2[F(1,0)+F(-1,0)] v\right\} \frac{\mathrm{d} v}{v^{2}}-2 \int_{-1}^{1} F(u, 0) \mathrm{d} u+\hat{H},
$$

where $F^{(1,2)}(u, v)$ and $F(u, 0)$ are defined by (42) and (44), respectively, and $\hat{H}$ is defined by (104). Expression (120) may be indicated, using a short algorithm-like notation, as

$$
I_{C}=\mathscr{C}(E, \psi, \varphi) .
$$

In the same fashion, the analysis of Sections 5 and 6 leads to the final formula for the adjacent double integration over the pair $\left(E, E^{\prime}\right)$ of elements joined by the common node $\boldsymbol{z}^{\prime}$, which stems from Eqs. (72) and (117) and reads:

$$
I_{\mathrm{A}}=I_{\mathrm{A}}^{(1)}+\int_{-1}^{1} \int_{0}^{1} \frac{1}{v}\left[Q_{\mathrm{A}}(u, v)-Q_{\mathrm{A}}(u, 0)\right] \mathrm{d} v \mathrm{~d} u+\int_{-1}^{1} Q_{\mathrm{A}}(u, 0) \ln s_{\mathrm{A}}(u) \mathrm{d} u+H^{+}-2 F(1,0) \ln \left(2 a^{+}\right),
$$

where $I_{\mathrm{A}}^{(1)}$ is the nonsingular integral over region 1 (see Section 5.1), $s_{\mathrm{A}}(u)$ and $Q_{\mathrm{A}}(u, v)$ are defined by $(63)$ and (66), respectively, while $H^{+}$is given by (118). Expression (122), in a short notation similar to (121), may be indicated as

$$
I_{\mathrm{A}}=\mathscr{A}\left(\{E, \psi\},\left\{E^{\prime}, \varphi^{\prime}\right\}\right) .
$$

Likewise, the final formula for the transposed adjacent double integration over the pair $\left(E^{\prime \prime}, E\right)$ is:

$$
I_{\mathrm{T}}=I_{\mathrm{T}}^{(2)}+\int_{-1}^{1} \int_{0}^{1} \frac{1}{v}\left[Q_{\mathrm{T}}(u, v)-Q_{\mathrm{T}}(u, 0)\right] \mathrm{d} v \mathrm{~d} u+\int_{-1}^{1} Q_{\mathrm{T}}(u, 0) \ln s_{\mathrm{T}}(u) \mathrm{d} u+H^{-}-2 F(-1,0) \ln \left(2 a^{-}\right),
$$

where $I_{\mathrm{T}}^{(2)}$ is the nonsingular integral over region 2 (see Section 5.2) while $s_{\mathrm{T}}(u), Q_{\mathrm{T}}(u, v)$ and $H^{-}$are defined by (77), (78) and (119), respectively. Similarly to (121) and (123), this result may be indicated as

$$
I_{\mathrm{T}}=\mathscr{T}\left(\{E, \psi\},\left\{E^{\prime \prime}, \varphi^{\prime \prime}\right\}\right) .
$$

Note that the nonintegral endpoint contributions to the coincident double integration in (56) have ultimately been considered as adjacent terms (and therefore appear in (122) and (124)), because having all element endpoint data (Jacobians, tangents and so on) used in the same program segment is simpler and computationally more efficient.

\section{Symmetry considerations}

In the symmetric Galerkin BEM, the unknown and weight functions $\varphi$ and $\psi$ are assumed to belong to the same function space; this implies here that $\psi$ has, like $\varphi$, interelement continuity. It is then useful to consider the effect of switching the roles of $\psi$ and $\varphi$ in the integration formulas.

First, from (31), (32), (42), (44) and (104), it is easy to see that

$$
F^{1}(u, v ; \varphi, \psi)=F^{2}(u, v ; \psi, \varphi), \quad F(u, 0 ; \varphi, \psi)=F(u, 0 ; \psi, \varphi),
$$




$$
\hat{H}(\psi, \varphi)=\hat{H}(\varphi, \psi),
$$

using obvious notations to emphasize the dependence in $\psi$ and $\varphi$. It follows that, with the notation (121), we have

$$
\mathscr{C}(E, \psi, \varphi)=\mathscr{C}(E, \varphi, \psi),
$$

i.e. the coincident double integration procedure is symmetric.

Next, consider the formulas $\mathscr{A}(\cdot, \cdot)$ and $\mathscr{T}(\cdot, \cdot)$ applied to the same consecutive pair of boundary elements $E, E^{\prime}$ (instead of the pair $E^{\prime \prime}, E$ for formula $\mathscr{T}(\cdot, \cdot)$ ). The functions $s_{\mathrm{A}}(u)$ and $s_{\mathrm{T}}(u)$ defined by (63) and (77) are readily found to be equal, and so are the vector functions $\boldsymbol{r}_{\mathrm{A}}(u)$ and $\boldsymbol{r}_{\mathrm{T}}(u)$ defined by (62) and (76) by virtue of (75). One then finds that

$$
Q_{\mathrm{A}}(u, v ; \varphi, \psi)=Q_{\mathrm{T}}(u, v ; \psi, \varphi) .
$$

This in turn implies that the integral terms of Eq. (124) with the roles of $\psi$ and $\varphi$ switched are equal to the corresponding integral terms of Eq. (122). On the other hand, the nonintegral terms in (122) are clearly symmetric in $\psi$ and $\varphi$, as are those of (124). These two contributions have the form $B_{\mathrm{A}} \psi\left(\boldsymbol{z}^{\prime}\right) \varphi\left(\boldsymbol{z}^{\prime}\right)$ and $B_{\mathrm{T}} \psi\left(\boldsymbol{z}^{\prime}\right) \varphi\left(\boldsymbol{z}^{\prime}\right)$, where $\boldsymbol{z}^{\prime}$ is the common endpoint of $E$ and $E^{\prime}$, and are not necessarily equal (i.e. $B_{\mathrm{A}} \neq B_{\mathrm{T}}$ ). However, the final Galerkin BEM system comes from summing all double element integrals, which implies that only the value of $B_{\mathrm{A}}+B_{\mathrm{T}}$ matters in the end. Therefore, one can freely replace $I_{\mathrm{A}}$ and $I_{\mathrm{T}}$ defined by (122) and (124) by:

$$
\begin{aligned}
& \tilde{I}_{\mathrm{A}}=I_{\mathrm{A}}^{(1)}+\int_{-1}^{1} \int_{0}^{1} \frac{1}{v}\left[Q_{\mathrm{A}}(u, v)-Q_{\mathrm{A}}(u, 0)\right] \mathrm{d} v \mathrm{~d} u+D_{\mathrm{A}} \psi(\boldsymbol{z}) \varphi(\boldsymbol{z}), \\
& \tilde{I}_{\mathrm{T}}=I_{\mathrm{T}}^{(2)}+\int_{-1}^{1} \int_{0}^{1} \frac{1}{v}\left[Q_{\mathrm{T}}(u, v)-Q_{\mathrm{T}}(u, 0)\right] \mathrm{d} v \mathrm{~d} u+D_{\mathrm{T}} \psi(z) \varphi(\boldsymbol{z})
\end{aligned}
$$

with

$$
\begin{aligned}
& D_{\mathrm{A}} \psi(\boldsymbol{z}) \varphi(\boldsymbol{z})=\int_{-1}^{1} Q_{\mathrm{A}}(u, 0) \ln s_{\mathrm{A}}(u) \mathrm{d} u+\frac{1}{2}\left(H^{+}(E)+H^{-}\left(E^{\prime}\right)\right)-F(1,0) \ln (2 a)-F^{\prime}(-1,0) \ln \left(2 a^{\prime}\right), \\
& D_{\mathrm{T}} \psi(\boldsymbol{z}) \varphi(\boldsymbol{z})=\int_{-1}^{1} Q_{\mathrm{T}}(u, 0) \ln s_{\mathrm{T}}(u) \mathrm{d} u+\frac{1}{2}\left(H^{+}(E)+H^{-}\left(E^{\prime}\right)\right)-F(1,0) \ln (2 a)-F^{\prime}(-1,0) \ln \left(2 a^{\prime}\right),
\end{aligned}
$$

where $a$ and $a^{\prime}$ denote the Jacobians of $E$ and $E^{\prime}$ at the shared endpoint $z$. Besides, from (118) and (119), one has:

$$
\begin{aligned}
H^{+}(E)+H^{-}\left(E^{\prime}\right)= & \mathscr{H}_{i}^{\prime}(-\pi, 0,1) n_{i}^{\prime}-\mathscr{H}_{i}(0,0,1) n_{i}-[\psi \varphi](1) \\
& \times \int_{0}^{\beta^{\prime}}\left\{\mathscr{H}_{i}(\theta, 0,1) n_{i}+\mathscr{H}_{i}(\pi-\theta, 0,1) n_{i}^{\prime}\right\} \frac{\cos \left(\theta-\beta^{\prime}\right)}{\sin \beta^{\prime}} \mathrm{d} \theta .
\end{aligned}
$$

This time, with the notations (123) and (125), we have:

$$
\tilde{\mathscr{A}}\left(\{E, \psi\},\left\{E^{\prime}, \varphi^{\prime}\right\}\right)=\tilde{\mathscr{T}}\left(\left\{E^{\prime}, \varphi^{\prime}\right\},\{E, \psi\}\right) .
$$

This observation has two important consequences. Firstly, it makes possible to code just one of the two procedures. Secondly, the formulas proposed for the adjacent cases are, like in (126), symmetry pre- 
serving even when affected by numerical integration errors. This feature may not be enjoyed by other regularization techniques (that is by all those methods that keep the distinction between inner and outer integration).

\section{Explicit expression of the free term}

In this section, explicit formulas are provided for the free term (129) established for the symmetric Galerkin BEM, as well as other quantities involved in the direct integration algorithm, for three cases: potential problems for isotropic and anisotropic media (Sections 9.1 and 9.2) and isotropic elasticity (Section 9.3).

\subsection{Isotropic potential problems}

The free-plane fundamental solution for the Laplace equation $\nabla^{2} \varphi=0$ is given by:

$$
\begin{aligned}
G(\boldsymbol{y}, \boldsymbol{x}) & =-\frac{1}{2 \pi} \ln r \\
T(\boldsymbol{y}, \boldsymbol{x}) & =-\frac{1}{2 \pi} \frac{\left(x_{j}-y_{j}\right) n_{j}(\boldsymbol{x})}{r^{2}}=W(\boldsymbol{x}, \boldsymbol{y}), \\
V_{i j}(\boldsymbol{y}, \boldsymbol{x}) & =\frac{1}{2 \pi}\left(\frac{\delta_{i j}}{r^{2}}-\frac{2\left(x_{i}-y_{i}\right)\left(x_{j}-y_{j}\right)}{r^{4}}\right) .
\end{aligned}
$$

The singular part $\mathscr{V}_{i j}$ of $V_{i j}$ is given by (9), repeated for convenience:

$$
\mathscr{V}_{i j}(\boldsymbol{e}, r, \boldsymbol{y})=\frac{1}{2 \pi}\left(\delta_{i j}-2 e_{i} e_{j}\right) .
$$

Explicit expressions can then readily be obtained for various related quantities involved in (120), (122) and (124). From (44), one finds that:

$$
F(u, 0)=\frac{1}{4 \pi} \psi(u) \varphi(u),
$$

while the function $\mathscr{H}_{i}(\omega, \varepsilon, \eta)$ defined by (90) is given by:

$$
\mathscr{H}_{i}(\omega, \varepsilon, \eta)=\frac{1}{2 \pi}\left[\sin \omega t_{i}(\eta)+(1-\cos \omega) n_{i}(\eta)\right] .
$$

Finally, since $\mathscr{V}_{i j}$ is symmetric in $(i, j)$, with reference to (104), one has $\hat{H}=0$.

Using (134) and (135), condition (104) for the cancellation of $\mathrm{O}\left(\varepsilon^{-1}\right)$ terms is readily verified. Besides, the practical evaluation of the free term (129) and the verification of condition (83) for the cancellation of $\mathrm{O}(\ln |\varepsilon|)$ terms are facilitated by the introduction of the polar angle $\omega$ through:

$$
\boldsymbol{e}_{\mathrm{A}}(u)=\cos \omega \boldsymbol{t}+\sin \omega \boldsymbol{n} \equiv \boldsymbol{e}_{\mathrm{A}}(\omega) \quad\left(0 \leqslant \omega \leqslant \beta^{\prime}\right) .
$$

Elementary calculations then yield:

$$
\begin{aligned}
\frac{\mathrm{d} u}{s_{\mathrm{A}}^{2}(u)} & =\frac{1}{2 a a^{\prime} \sin \beta^{\prime}} \mathrm{d} \omega, \\
s_{\mathrm{A}}(u) & =\frac{2 a a^{\prime} \sin \beta^{\prime}}{a \sin \omega-a^{\prime} \sin \left(\omega-\beta^{\prime}\right)} \equiv \tilde{s}_{\mathrm{A}}(\omega)
\end{aligned}
$$


and $Q_{\mathrm{A}}(u, 0)$ defined by $(68)$ becomes:

$$
Q_{\mathrm{A}}(u, 0) \mathrm{d} u=\frac{1}{2 \pi} \frac{\cos \left(2 \omega-\beta^{\prime}\right)}{\sin \beta^{\prime}} \psi \varphi^{\prime} \mathrm{d} \omega .
$$

Using these definitions, condition (83) for the cancellation of $\mathrm{O}(\ln |\varepsilon|)$ terms takes the form:

$$
\frac{1}{2 \pi}=\frac{1}{2 \pi} \int_{0}^{\beta^{\prime}} \frac{\cos \left(2 \omega-\beta^{\prime}\right)}{\sin \beta^{\prime}} \mathrm{d} \omega
$$

and is readily checked via a direct calculation, while the free term (129) becomes:

$$
D_{\mathrm{A}}=\frac{1}{2 \pi} \int_{0}^{\beta^{\prime}}\left\{\frac{\cos \left(2 \omega-\beta^{\prime}\right)}{\sin \beta^{\prime}} \ln \left|\tilde{s}_{\mathrm{A}}(\omega)\right|-\frac{\cos \omega \cos \left(\omega-\beta^{\prime}\right)}{\sin \beta^{\prime}}\right\} \mathrm{d} \omega+\frac{1}{4 \pi}\left(2-\ln 4 a a^{\prime}\right) .
$$

Upon performing an analytical integration, one finds (having put $\chi=a^{\prime} / a$ ):

$$
D_{\mathrm{A}}=\frac{1}{4 \pi}\left[2+\frac{\left(1-\chi^{2}\right) \ln \chi-2 \chi \beta^{\prime} \sin \beta^{\prime}}{1+\chi^{2}-2 \chi \cos \beta^{\prime}}\right] \text {. }
$$

\subsection{2-D anisotropic potential problems}

The medium is now assumed to have anisotropic constitutive properties, characterized by a positive definite second-order tensor $\boldsymbol{K}$ with coefficients $k_{1}, k_{2}>0$ and orthogonal unit vectors $\boldsymbol{w}_{1}, \boldsymbol{w}_{2}$ such that:

$$
\boldsymbol{K}=k_{1} \boldsymbol{w}_{1} \otimes \boldsymbol{w}_{1}+k_{2} \boldsymbol{w}_{2} \otimes \boldsymbol{w}_{2} .
$$

The flux $q$ associated to the potential $\varphi$ is now given by:

$$
q=(\boldsymbol{K} \cdot \nabla u) \cdot \boldsymbol{n}=k_{i j} \varphi_{, j} n_{i} .
$$

In that case, the free plane fundamental solution $G(\boldsymbol{y}, \boldsymbol{x})$ solves $\boldsymbol{\nabla} \cdot(\boldsymbol{K} \cdot \nabla \varphi)+\delta(\boldsymbol{x}-\boldsymbol{y})=0$ and is given by:

$$
\begin{aligned}
G(\boldsymbol{y}, \boldsymbol{x}) & =-\frac{1}{4 \pi \Delta} \ln R^{2}, \\
T(\boldsymbol{y}, \boldsymbol{x}) & =-\frac{1}{2 \pi \Delta} \frac{r_{j} n_{j}(\boldsymbol{x})}{R^{2}}=W(\boldsymbol{x}, \boldsymbol{y}), \\
V_{i j}(\boldsymbol{y}, \boldsymbol{x}) & =\frac{1}{2 \pi \Delta}\left[\frac{1}{R^{2}} K_{i j}-\frac{2 r_{i} r_{j}}{R^{4}}\right],
\end{aligned}
$$

where $\Delta=\left(k_{1} k_{2}\right)^{1 / 2}$ and $R$ is defined in terms of the position vector $\boldsymbol{r}$ by:

$$
R^{2}=\boldsymbol{r} \cdot \boldsymbol{K}^{-1} \cdot \boldsymbol{r}
$$

The singular factor of the hypersingular kernel $V(\boldsymbol{y}, \boldsymbol{x})$ is thus given by:

$$
\mathscr{V}_{i j}(\boldsymbol{e}, r, \boldsymbol{y})=\frac{1}{2 \pi \Delta}\left[\frac{1}{\left(\boldsymbol{e} \cdot \boldsymbol{K}^{-1} \cdot \boldsymbol{e}\right)} K_{i j}-\frac{2 e_{i} e_{j}}{\left(\boldsymbol{e} \cdot \boldsymbol{K}^{-1} \cdot \boldsymbol{e}\right)^{2}}\right] .
$$

Explicit expressions can then readily be obtained for various related quantities. From (44), one finds that:

$$
F(u, 0)=\frac{1}{4 \pi \Delta} \frac{\boldsymbol{n} \cdot \boldsymbol{K} \cdot \boldsymbol{n}}{\boldsymbol{e} \cdot \boldsymbol{K}^{-1} \cdot \boldsymbol{e}} \psi(u) \varphi(u)
$$

while the function $\mathscr{H}_{i}(\omega, \eta)$ defined by $(90)$ is readily found to be given by: 


$$
\mathscr{H}_{i}(\omega, \varepsilon, \eta)=\frac{1}{2 \pi \Delta}\left\{\frac{(\boldsymbol{K} \cdot \boldsymbol{d})_{i}}{\boldsymbol{e} \cdot \boldsymbol{K}^{-1} \cdot \boldsymbol{e}}+\frac{(\boldsymbol{K} \cdot \boldsymbol{n})_{i}}{\boldsymbol{t} \cdot \boldsymbol{K}^{-1} \cdot \boldsymbol{t}}\right\}
$$

where $d_{1}=e_{2}$ and $d_{2}=-e_{1}$. Using (144) and (145), condition (105) for the cancellation of $\mathrm{O}\left(\varepsilon^{-1}\right)$ terms is readily verified. Besides, utilizing again (136)-(138), $Q_{\mathrm{A}}(u, 0)$ defined by $(68)$ takes the form:

$$
Q_{\mathrm{A}}(u, 0) \mathrm{d} u=\frac{1}{2 \pi \Delta \sin \beta^{\prime}}\left[\frac{\boldsymbol{n} \cdot \boldsymbol{K} \cdot \boldsymbol{n}^{\prime}}{\boldsymbol{e} \cdot \boldsymbol{K}^{-1} \cdot \boldsymbol{e}}-\frac{2 \sin \omega \sin \left(\omega-\beta^{\prime}\right)}{\left(\boldsymbol{e} \cdot \boldsymbol{K}^{-1} \cdot \boldsymbol{e}\right)^{2}}\right] \mathrm{d} \omega
$$

and condition (83) for the cancellation of $\mathrm{O}(\ln |\varepsilon|)$ terms can again be checked via a direct analytic calculation. The free term (129) can also be calculated explicitly by analytic integration, and is given by:

$$
\begin{aligned}
D_{\mathrm{A}}= & \frac{\Delta}{16 \pi}\left[\frac{\kappa+\kappa^{-1}}{\bar{D}}\left(1-\chi^{2}\right)+\frac{\kappa-\kappa^{-1}}{\bar{D}}\left(\chi^{2} \cos 2\left(\gamma-\beta^{\prime}\right)-\cos 2 \gamma\right)\right] \ln \left(\chi^{2} \frac{D\left(\beta^{\prime}\right)}{D(0)}\right) \\
& -\frac{\Delta}{2 \pi} \frac{\chi \sin \beta^{\prime}}{\bar{D}}\left[\tan ^{-1}\left(\kappa \tan \left(\beta^{\prime}-\gamma\right)+\tan ^{-1}(\kappa \tan \gamma)\right)\right]+\frac{\Delta}{2 \pi},
\end{aligned}
$$

where $\kappa=\left(k_{1} / k_{2}\right)^{1 / 2}, \gamma=\left(\boldsymbol{t}, \boldsymbol{w}_{1}\right), D(\theta)=\kappa^{-1} \cos ^{2}(\theta-\gamma)+\kappa \sin ^{2}(\theta-\gamma)$ and $\bar{D}$ is given by (having again put $\left.\chi=a^{\prime} / a\right)$ :

$$
\bar{D}=D(0)-2 \chi D\left(\beta^{\prime} / 2\right)+\chi^{2} D\left(\beta^{\prime}\right)+\left(\kappa+\kappa^{-1}\right)\left(1-\cos \beta^{\prime}\right) .
$$

\subsection{2-D isotropic elasticity}

The counterpart of the starting identity (18) for plane elasticity is:

$$
\begin{aligned}
0=\int_{\Gamma} v_{k}(\boldsymbol{y})\left\{u_{\ell}(\boldsymbol{y}) \int_{s_{\varepsilon}} V_{k \ell}(\boldsymbol{y}, \boldsymbol{x}) \mathrm{d} s_{x}+\int_{s_{\varepsilon}}\left[u_{\ell, m}(\boldsymbol{y})\left(x_{m}-y_{m}\right) V_{k \ell}(\boldsymbol{y}, \boldsymbol{x})-\sigma_{\ell m}(\boldsymbol{y}) n_{m}(\boldsymbol{x}) W_{k \ell}(\boldsymbol{y}, \boldsymbol{x})\right] \mathrm{d} s_{x}\right. \\
\left.\quad+\int_{\Gamma-e_{\varepsilon}}\left\{V_{k \ell}(\boldsymbol{y}, \boldsymbol{x}) u_{\ell}(\boldsymbol{x})-W_{k \ell}(\boldsymbol{y}, \boldsymbol{x}) p_{\ell}(\boldsymbol{x})\right\} \mathrm{d} s_{x}\right\} \mathrm{d} s_{y}+\mathrm{O}(\varepsilon),
\end{aligned}
$$

where $\boldsymbol{u}$ and $\boldsymbol{\sigma}$ and $\boldsymbol{p}$ are displacements, stresses and tractions, respectively, and the kernels $V_{k \ell}(\boldsymbol{y}, \boldsymbol{x})$ and $W_{k \ell}(\boldsymbol{y}, \boldsymbol{x})$ (which are hypersingular and strongly singular, respectively) derive from the elastostatic fundamental solution. Eq. (148) is, in weighted-residual form with a trial displacement $\boldsymbol{v}$, the integral statement used as a starting point to obtain traction BIEs.

In particular, for $2 \mathrm{D}$ plane strain and isotropic elasticity, the hypersingular kernel $V_{k \ell}(\boldsymbol{y}, \boldsymbol{x})$ is given by:

$$
\begin{aligned}
& V_{k \ell}(\boldsymbol{y}, \boldsymbol{x})=V_{k \ell i j}(\boldsymbol{y}, \boldsymbol{x}) n_{i}(\boldsymbol{y}) n_{j}(\boldsymbol{x})=\frac{1}{r^{2}} \mathscr{V}_{k \ell i j}(\boldsymbol{y}, \boldsymbol{x}) n_{i}(\boldsymbol{y}) n_{j}(\boldsymbol{x}) \\
& \mathscr{V}_{k \ell i j}(\boldsymbol{y}, \boldsymbol{x})=A\left[2(1-2 v)\left[\delta_{i j} \delta_{k \ell}+\delta_{j k} \delta_{i \ell}+2 \delta_{i k} e_{j} e_{\ell}+2 \delta_{j \ell} e_{i} e_{k}\right]\right. \\
& \left.\quad+4 v\left[\delta_{i j} e_{k} e_{\ell}+\delta_{k \ell} e_{i} e_{j}+\delta_{i \ell} e_{j} e_{k}+\delta_{j k} e_{i} e_{\ell}\right]+(8 v-2) \delta_{i k} \delta_{j \ell}-16 e_{i} e_{j} e_{k} e_{\ell}\right]
\end{aligned}
$$

where $A=\mu /(4 \pi(1-v))$. Explicit expressions are then readily obtained for related quantities. From (44), one finds that:

$$
F(u, 0)=A u_{i}(u) v_{i}(u),
$$

while the function $\mathscr{H}_{i j k}(\omega, \varepsilon, \eta)$ defined by (90) is given by:

$$
\mathscr{H}_{k \ell i}(\omega, \varepsilon, \eta)=2 A\left[\delta_{k \ell} d_{i}+\delta_{\ell i} d_{k}+\delta_{k i} d_{\ell}-2 d_{k} d_{\ell} d_{i}\right],
$$


where, once again, $d_{1}=e_{2}$ and $d_{2}=-e_{1}$. Using (151) and (152), condition (105) for the cancellation of $\mathrm{O}\left(\varepsilon^{-1}\right)$ terms is readily verified. Besides, utilizing again (136)-(138), $Q_{\mathrm{A} ; k \ell}(u, 0)$ defined by (68) takes the form:

$$
\begin{aligned}
Q_{\mathrm{A} ; \mathrm{k} \ell}(u, 0) \mathrm{d} u=[ & {\left[2 \cos \left(4 \omega-\beta^{\prime}\right)-\cos \left(2 \omega-\beta^{\prime}\right)-\cos \left(2 \omega+\beta^{\prime}\right)\right]\left(t_{k} t_{\ell}-n_{k} n_{\ell}\right) } \\
+ & {\left[2 \sin \left(4 \omega-\beta^{\prime}\right)-\sin \left(2 \omega-\beta^{\prime}\right)-\sin \left(2 \omega+\beta^{\prime}\right)\right]\left(t_{k} n_{\ell}+n_{k} t_{\ell}\right) } \\
& \left.+2 \cos \left(2 \omega-\beta^{\prime}\right) \delta_{k \ell}\right] \frac{A \mathrm{~d} \omega}{\sin \beta^{\prime}} .
\end{aligned}
$$

Using these definitions, condition (83) for the cancellation of $\mathrm{O}(\ln |\varepsilon|)$ terms is once again readily checked via a direct calculation. The free term (129), also explicitly calculable by analytic integration, is given by:

$$
\begin{aligned}
D_{A ; k \ell}=A & \left\{\left[2+\frac{1-\chi^{2}}{\Xi} \ln \chi-\frac{2 \chi \sin \beta^{\prime}}{\Xi} \beta^{\prime}\right] \delta_{k \ell}+\frac{1-\chi^{2}}{\Xi}\left[-\frac{2 \chi \sin ^{2} \beta^{\prime}}{\Xi}\left(t_{k} t_{\ell}^{\prime}-n_{k} n_{\ell}^{\prime}\right) \ln \chi\right.\right. \\
& \left.\left.+\frac{2 \chi \sin ^{2} \beta^{\prime}}{\Xi} \beta^{\prime}-\sin \beta^{\prime}\right)\left(t_{k} n_{\ell}^{\prime}+n_{k} t_{\ell}^{\prime}\right)\right]-\frac{2 \chi \sin \beta^{\prime}}{\Xi}\left[\frac{2 \chi-\left(1+\chi^{2}\right) \cos \beta^{\prime}}{\Xi} \ln \chi\left(t_{k} n_{\ell}^{\prime}+n_{k} t_{\ell}^{\prime}\right)\right. \\
& \left.\left.\left.+\frac{2 \chi-\left(1+\chi^{2}\right) \cos \beta^{\prime}}{\Xi} \beta^{\prime}+\sin \beta^{\prime}\right)\left(t_{k} t_{\ell}^{\prime}-n_{k} n_{\ell}^{\prime}\right)\right]\right\}
\end{aligned}
$$

having put $\Xi=1+\chi^{2}-2 \chi \cos \beta^{\prime}$.

\section{Numerical tests}

A MATLAB program has been written to test the present direct integration method, that is, Eqs. (120), (127) and (128). To demonstrate its accuracy, we present a comparison between numerical and exact (analytical) values of double hypersingular integrals of the form

$$
I=\lim _{\varepsilon \rightarrow 0}\left\{\int_{E_{1}+E_{2}} \int_{E_{1}+E_{2}-e_{\varepsilon}} V(\boldsymbol{y}, \boldsymbol{x}) \varphi_{1}(\boldsymbol{y}) \varphi_{2}(\boldsymbol{x}) \mathrm{d} s_{x} \mathrm{~d} s_{y}\right\},
$$

where $V(\boldsymbol{y}, \boldsymbol{x})=V_{i j}(\boldsymbol{y}, \boldsymbol{x}) n_{i}(\boldsymbol{y}) n_{j}(\boldsymbol{x})$ with $V_{i j}(\boldsymbol{y}, \boldsymbol{x})$ defined by (133), $E_{1}, E_{2}$ are straight boundary elements meeting at their common endpoint with an angle $\beta$ (Fig. 8), and $\varphi_{1}$ and $\varphi_{2}$ are taken as the same piecewise linear 'hat function' in $s$

$$
\left(\varphi_{1}, \varphi_{2}\right)(s)=\frac{1+s}{2}\left(\text { on } E_{1}\right) \text { and }\left(\varphi_{1}, \varphi_{2}\right)(s)=\frac{1-s}{2}\left(\text { on } E_{2}\right),
$$

where $s$ is a curvilinear abscissa ranging from -1 to 1 on both elements.

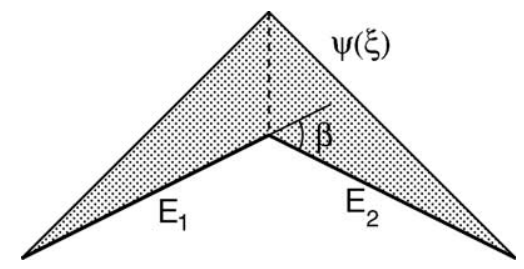

Fig. 8. Adjacent straight boundary elements $E_{1}, E_{2}$ and the 'hat function'. 
Integral $I$ in (154) is scale-independent (this stems from the fact that the kernel $V(\boldsymbol{y}, \boldsymbol{x})$ is homogeneous of degree -2 with respect to the position vector $\boldsymbol{x}-\boldsymbol{y}$, and hence depends only on $\beta$ and the element length ratio $\chi=\left|E_{1}\right| /\left|E_{2}\right|$ ). The exact value of (154) (in the limiting case as $\varepsilon \rightarrow 0$ ), including the free term, is found to be

$$
\begin{aligned}
I=\frac{1}{4 \pi} & \left\{-\left[2+\left(\chi+\chi^{-1}\right) \cos \beta\right] \ln \left(2 \cos \beta+\chi+\chi^{-1}\right)+\left(\chi-\chi^{-1}\right) \cos \beta \ln \chi\right. \\
& \left.+2 \sin \beta\left[\left(\chi+\chi^{-1}\right) \tan ^{-1}(\cot \beta)-\chi \tan ^{-1}\left(\cot \beta+\frac{\chi^{-1}}{\sin \beta}\right)-\chi^{-1} \tan ^{-1}\left(\cot \beta+\frac{\chi}{\sin \beta}\right)\right]\right\}
\end{aligned}
$$

Each straight element $E_{k}$ has been represented as a three-node quadratic element as in Eq. (22), with the inner node located at $s=d_{k}$. Therefore, only when $d_{k}=0$ we have the classical element with constant Jacobian, that is with a linear mapping $s=s(\xi)$. Any other value of $d_{k}$, although maintaining the element straight, does introduce a distortion in the mapping $s=s(\xi)$, making the numerical evaluation more difficult. The distortion parameters $d_{k}$ may vary in the range $[-0.5,0.5]$; with the extreme values we have the well known quarter-point element, often used in crack problems.

Since we wish the analytical value of the integral $I$ not to be affected by the distortion parameters $d_{k}$, we have to represent the functions $\varphi_{1}(s(\xi))=\varphi_{1}(\xi)$ and $\varphi_{2}(s(\xi))=\varphi_{2}(\xi)$ as quadratic functions in $\xi$ with nodal values

$$
\left\{0, \frac{1-d_{1}}{2}, 1\right\} \text { on } E_{1} \text { and }\left\{1, \frac{1+d_{2}}{2}, 0\right\} \text { on } E_{2} .
$$

Although it may seem a simple test, it is in fact quite demanding when $d_{k} \neq 0$ and $\beta \neq 0$, and could very well be considered like a benchmark to test methods for the evaluation of hypersingular integrals in the symmetric Galerkin BEM. Indeed, straight elements, when represented by means of nonlinear mappings, are as general as curved elements if the computation is performed in terms of the parametric coordinates, while exact values are available for comparison.

In particular we present here numerical results for $\chi=1$, that is elements of the same length, and $\chi=0.25$, which is beyond what is generally recommended in a well designed mesh. In both cases, the corner angle $\beta$ is taken equal to $0, \pi / 4, \pi / 2$ and $3 \pi / 4$, that is from smooth boundary up to an acute angle (usually avoided in applications). Moreover, to test the sensitivity of the proposed technique to the element distortion, we also consider the undistorted case $\left(d_{1}=d_{2}=0\right)$, the case in which $E_{1}$ is a quarter node element and $E_{2}$ is as before $\left(d_{1}=0.5, d_{2}=0\right)$, and the case in which both elements are quarter point $\left(d_{1}=0.5, d_{2}=\right.$ $-0.5)$ and hence exhibit the maximum degree of distortion.

The analytical values of the integral $I$, computed by means of Eq. (155), are as shown in Table 1. Of course the distortions do not affect the exact values.

The relative errors for the case $\chi=1$, i.e. two elements of equal length, are given in Table 2 . All integrals have been computed using Gauss formulas with $n_{G}$ points for one-dimensional integrals and $n_{G} \times n_{G}$ points for double ones. We see from Table 2 that even a severe distortion of the elements does not have much influence on the accuracy of the results. On the other hand, the higher the angle $\beta$, the more demanding is the numerical computation.

Table 1

Exact values of the integral $I$ for several values of the element length ratio $\chi$ and of the corner angle $\beta$

\begin{tabular}{lllll}
\hline$\chi$ & $\beta=0^{\circ}$ & $\beta=45^{\circ}$ & $\beta=90^{\circ}$ & $\beta=135^{\circ}$ \\
\hline 0 & -5.545177444479 & -5.303194641153 & -4.527887014709 & -3.018883618685 \\
0.25 & -6.255030294227 & -6.072331681256 & -5.516576102721 & -4.583045906354 \\
\hline
\end{tabular}


Table 2

Relative errors in the case $\chi=1$, for several numbers $n_{G}$ of Gauss points

\begin{tabular}{|c|c|c|c|c|c|}
\hline & $n_{G}$ & $\beta=0^{\circ}$ & $\beta=45^{\circ}$ & $\beta=90^{\circ}$ & $\beta=135^{\circ}$ \\
\hline \multirow[t]{5}{*}{$d_{1}=d_{2}=0$} & 4 & $3.5 \mathrm{E}-06$ & $-5.8 \mathrm{E}-06$ & $-9.2 \mathrm{E}-03$ & $-8.4 \mathrm{E}-01$ \\
\hline & 8 & $5.9 \mathrm{E}-12$ & $-2.2 \mathrm{E}-11$ & $-1.5 \mathrm{E}-05$ & $-6.7 \mathrm{E}-02$ \\
\hline & 12 & $1.5 \mathrm{E}-13$ & $1.6 \mathrm{E}-13$ & $-1.9 \mathrm{E}-08$ & $-3.9 \mathrm{E}-03$ \\
\hline & 16 & $-1.2 \mathrm{E}-12$ & $-1.3 \mathrm{E}-12$ & $-2.3 \mathrm{E}-11$ & $-2.0 \mathrm{E}-04$ \\
\hline & 20 & $2.0 \mathrm{E}-12$ & $2.1 \mathrm{E}-12$ & $2.4 \mathrm{E}-12$ & $-1.0 \mathrm{E}-05$ \\
\hline \multirow[t]{5}{*}{$d_{1}=0.5, d_{2}=0$} & 4 & $3.0 \mathrm{E}-07$ & $-2.5 \mathrm{E}-04$ & $2.3 \mathrm{E}-02$ & $1.7 \mathrm{E}+00$ \\
\hline & 8 & $-2.6 \mathrm{E}-08$ & $-4.1 \mathrm{E}-08$ & $-3.4 \mathrm{E}-05$ & $-1.8 \mathrm{E}-01$ \\
\hline & 12 & $-1.6 \mathrm{E}-09$ & $-1.6 \mathrm{E}-09$ & $-9.6 \mathrm{E}-08$ & $1.4 \mathrm{E}-02$ \\
\hline & 16 & $-1.9 \mathrm{E}-10$ & $-2.0 \mathrm{E}-10$ & $3.5 \mathrm{E}-10$ & $-1.0 \mathrm{E}-03$ \\
\hline & 20 & $-3.4 \mathrm{E}-11$ & $-3.6 \mathrm{E}-11$ & $-4.4 \mathrm{E}-11$ & $5.9 \mathrm{E}-05$ \\
\hline \multirow[t]{5}{*}{$d_{1}=0.5, d_{2}=-0.5$} & 4 & $-5.7 \mathrm{E}-06$ & $-9.4 \mathrm{E}-05$ & $-2.6 \mathrm{E}-02$ & $-1.7 \mathrm{E}+00$ \\
\hline & 8 & $-5.4 \mathrm{E}-08$ & $-5.3 \mathrm{E}-08$ & $-5.3 \mathrm{E}-05$ & $-1.5 \mathrm{E}-01$ \\
\hline & 12 & $-3.2 \mathrm{E}-09$ & $-3.3 \mathrm{E}-09$ & $-8.0 \mathrm{E}-08$ & $-1.0 \mathrm{E}-02$ \\
\hline & 16 & $-3.9 \mathrm{E}-10$ & $-4.0 \mathrm{E}-10$ & $-5.7 \mathrm{E}-10$ & $-5.8 \mathrm{E}-04$ \\
\hline & 20 & $-7.1 \mathrm{E}-11$ & $-7.4 \mathrm{E}-11$ & $-8.7 \mathrm{E}-11$ & $-3.0 \mathrm{E}-05$ \\
\hline
\end{tabular}

The relative errors for the case $\chi=0.25$ are provided in Table 3 for the same values of the corner angle and of the distortion parameters. Table 3 is quite similar to Table 2, thus showing that the algorithm can cope very well with adjacent elements of very different length.

To further validate the present direct approach to SGBEM, and in particular to emphasize the consistency and correctness of the overall SGBEM formulation, a sample two-dimensional potential problem is finally considered. Let $\Omega$ be the bounded region enclosed in the ellipse of equation $x_{1}^{2}+\left(x_{2} / a\right)^{2}-1=0$. The function $\varphi=x^{3}-3 x y^{2}$ solves the Laplace equation $\nabla^{2} \varphi=0$ and is associated with the normal derivative

$$
q=\frac{\partial \varphi}{\partial n}=\frac{3 x_{1}\left[\left(x_{1}^{2}-x_{2}^{2}\right) a^{2}-2 x_{2}^{2}\right]}{\left(x_{1}^{2} a^{4}+x_{2}^{2}\right)^{1 / 2}} \quad\left(x_{1}, x_{2}\right) \in \partial \Omega
$$

Table 3

Relative errors in the case $\chi=0.25$

\begin{tabular}{|c|c|c|c|c|c|}
\hline & $n_{G}$ & $\beta=0^{\circ}$ & $\beta=45^{\circ}$ & $\beta=90^{\circ}$ & $\beta=135^{\circ}$ \\
\hline \multirow[t]{5}{*}{$d_{1}=d_{2}=0$} & 4 & $-1.3 \mathrm{E}-03$ & $4.0 \mathrm{E}-03$ & $-4.5 \mathrm{E}-02$ & $-9.1 \mathrm{E}-01$ \\
\hline & 8 & $-4.0 \mathrm{E}-07$ & $-2.3 \mathrm{E}-06$ & $-4.1 \mathrm{E}-04$ & $-6.5 \mathrm{E}-02$ \\
\hline & 12 & $-9.1 \mathrm{E}-11$ & $-1.2 \mathrm{E}-10$ & $-2.5 \mathrm{E}-06$ & $7.9 \mathrm{E}-03$ \\
\hline & 16 & $-1.1 \mathrm{E}-12$ & $1.9 \mathrm{E}-13$ & $-1.0 \mathrm{E}-08$ & $1.9 \mathrm{E}-03$ \\
\hline & 20 & $1.8 \mathrm{E}-12$ & $1.9 \mathrm{E}-12$ & $-2.9 \mathrm{E}-13$ & $1.7 \mathrm{E}-04$ \\
\hline \multirow[t]{5}{*}{$d_{1}=0.5, d_{2}=0$} & 4 & $1.0 \mathrm{E}-05$ & $-2.6 \mathrm{E}-04$ & $2.7 \mathrm{E}-02$ & $1.3 \mathrm{E}+00$ \\
\hline & 8 & $-2.6 \mathrm{E}-08$ & $-3.4 \mathrm{E}-08$ & $-8.3 \mathrm{E}-05$ & $-1.2 \mathrm{E}-01$ \\
\hline & 12 & $-1.4 \mathrm{E}-09$ & $-1.4 \mathrm{E}-09$ & $4.3 \mathrm{E}-08$ & $8.4 \mathrm{E}-03$ \\
\hline & 16 & $-1.7 \mathrm{E}-10$ & $-1.7 \mathrm{E}-10$ & $1.5 \mathrm{E}-10$ & $-4.4 \mathrm{E}-04$ \\
\hline & 20 & $-3.0 \mathrm{E}-11$ & $-3.1 \mathrm{E}-11$ & $-3.6 \mathrm{E}-11$ & $1.7 \mathrm{E}-05$ \\
\hline \multirow{5}{*}{$d_{1}=0.5, d_{2}=-0.5$} & 4 & $-5.6 \mathrm{E}-04$ & $3.2 \mathrm{E}-03$ & $-9.8 \mathrm{E}-02$ & $-1.5 \mathrm{E}+00$ \\
\hline & 8 & $1.1 \mathrm{E}-06$ & $5.9 \mathrm{E}-06$ & $-1.4 \mathrm{E}-03$ & $-1.5 \mathrm{E}-02$ \\
\hline & 12 & $-1.2 \mathrm{E}-09$ & $-1.5 \mathrm{E}-08$ & $-1.4 \mathrm{E}-05$ & $3.5 \mathrm{E}-02$ \\
\hline & 16 & $-3.4 \mathrm{E}-10$ & $-3.5 \mathrm{E}-10$ & $-9.6 \mathrm{E}-08$ & $5.5 \mathrm{E}-03$ \\
\hline & 20 & $-6.3 \mathrm{E}-11$ & $-6.5 \mathrm{E}-11$ & $-6.4 \mathrm{E}-10$ & $3.4 \mathrm{E}-04$ \\
\hline
\end{tabular}


Table 4

Neumann problem on an ellipse: relative mean squared errors on the solution

\begin{tabular}{llll}
\hline Number of elements & 40 & 80 & 160 \\
\hline$a=0.25$ & $4.13 \mathrm{E}-3$ & $5.99 \mathrm{E}-4$ & $1.00 \mathrm{E}-4$ \\
$a=0.50$ & $6.08 \mathrm{E}-3$ & $1.01 \mathrm{E}-3$ & $2.05 \mathrm{E}-4$ \\
$a=1.00$ & $7.98 \mathrm{E}-3$ & $1.36 \mathrm{E}-3$ & $2.81 \mathrm{E}-4$ \\
$a=2.00$ & $1.17 \mathrm{E}-2$ & $2.57 \mathrm{E}-3$ & $6.15 \mathrm{E}-4$ \\
$a=4.00$ & $3.99 \mathrm{E}-2$ & $9.92 \mathrm{E}-3$ & $2.48 \mathrm{E}-3$ \\
\hline
\end{tabular}

The Neumann problem has been solved numerically using the limiting form of the weighted hypersingular integral equation (13). The relative mean squared errors obtained on the nodal values of $\phi$ for three mesh sizes (40, 80 and 160 three-noded boundary elements respectively) and several aspects ratios $a$ of the ellipse are presented in Table 4.

\section{Conclusions}

In this paper the direct approach has been extended to the evaluation of double hypersingular integrals like those arising in the symmetric Galerkin BEM. Double integrals are considered as a whole through the introduction of suitable coordinate transformations in the two-dimensional space of intrinsic coordinates. As a result, the proposed procedure preserves the symmetry of the formulation after discretization, even when the numerical quadratures are affected by some errors.

As typical in the direct method, the limiting process has been expressed in the parametric space and the free terms have been evaluated analytically. Cancellation of all potentially unbounded terms has been shown to occur if the density function $\varphi$ is continuous between elements. The analysis has also shown that somehow new free terms arise.

Numerical tests have shown that the algorithm behaves very well even when adjacent elements have different length and have nonconstant Jacobians. The effect of the angle between elements has been also pointed out.

\section{Acknowledgement}

Part of this work was carried on while M. Guiggiani was visiting the Ecole Polytechnique as maittre de recherche. The support is gratefully acknowledged.

\section{References}

[1] S. Sirtori, General stress analysis method by means of integral equations and boundary elements, Meccanica 14 (1979) $210-218$.

[2] G. Maier, C. Polizzotto, A Galerkin approach to boundary element elastoplastic analysis, Comp. Methods Appl. Mech. Engrg. 60 (1987) 175-194.

[3] M. Bonnet, G. Maier, C. Polizzotto, Symmetric Galerkin boundary element method, Appl. Mech. Rev. 51 (1998) 669-704.

[4] J.H. Kane, C. Balakrishna, Symmetric Galerkin boundary formulations employing curved elements, Int. J. Num. Methods Engrg. 36 (1993) 2157-2187.

[5] M. Bonnet, Regularized direct and indirect symmetric variational BIE formulations for three-dimensional elasticity, Engrg. Anal. Bound. Elem. 15 (1995) 93-102.

[6] S. Sirtori, G. Maier, G. Novati, S. Miccoli, A Galerkin symmetric boundary element method in elasticity: formulation and implementation, Int. J. Num. Methods Engrg. 35 (1992) 255-282. 
[7] A. Frangi, G. Novati, Symmetric BE method in two-dimensional elasticity: evaluation of double integrals for curved elements, Comp. Mech. 19 (1996) 58-68.

[8] A. Frangi, M. Bonnet, A Galerkin symmetric and direct BIE method for Kirchhoff elastic plates: formulation and implementation, Int. J. Num. Methods Engrg. 41 (1998) 337-369.

[9] M. Bonnet, Boundary Integral Equations Methods for Solids and Fluids, John Wiley and Sons, 1999.

[10] C. Balakrishna, L.J. Gray, J.H. Kane, Efficient analytical integration of symmetric Galerkin boundary integrals over curved elements: thermal conduction formulation, Comp. Methods Appl. Mech. Engrg. 111 (1994) 335-355.

[11] C. Balakrishna, L.J. Gray, J.H. Kane, Efficient analytical integration of symmetric Galerkin boundary integrals over curved elements: elasticity formulation, Comp. Methods Appl. Mech. Engrg. 117 (1994) 157-179.

[12] L. Gray, Evaluation of singular and hypersingular Galerkin integrals: direct limits and symbolic computation, in: V. Sladek, J. Sladek (Eds.), Singular Integrals in Boundary Element Methods, Comp. Mech. Publ, Southampton, 1998, pp. 45-84, Ch. 2.

[13] A. Aimi, M. Diligenti, G. Monegato, New numerical integration schemes for applications of Galerkin BEM to 2-D problems, Int. J. Num. Methods Engrg. 40 (1997) 1977-1999.

[14] A. Aimi, M. Diligenti, G. Monegato, Numerical integration schemes for the BEM solution of hypersingular integral equations, Int. J. Num. Methods Engrg. 45 (1999) 1807-1830.

[15] A. Carini, M. Diligenti, P. Maranesi, M. Zanella, Analytical integrations for two-dimensional elastic analysis by the symmetric Galerkin boundary element method, Comp. Mech. 23 (1999) 308-323.

[16] W. Hackbush, S.A. Sauter, On the efficient use of the Galerkin method to solve Fredholm integral equation, Appl. Math. 38 (1993) 301-322.

[17] T. von Petersdorff, C. Schwab, Fully discrete multiscale Galerkin BEM, Tech. Rep. 95-08, ETH, Zurich, Switzerland, 1995.

[18] S.A. Sauter, C. Schwab, Quadrature for $h p$-Galerkin BEM in $R^{3}$, Num. Math. 78 (1997) 211-258.

[19] S. Erichsen, S.A. Sauter, Efficient automatic quadrature in 3-D Galerkin BEM, Comp. Methods Appl. Mech. Engrg. 157 (1998) 215-224.

[20] H. Andrä, E. Schnack, Integration of singular Galerkin-type boundary element integrals for 3D elasticity problems, Num. Math. 76 (1997) 143-165.

[21] H. Andrä, Integration of singular integrals for the Galerkin-type boundary element method in 3D elasticity, Comp. Methods Appl. Mech. Engrg. 157 (1998) 239-249.

[22] M. Guiggiani, A. Gigante, A general algorithm for multidimensional Cauchy principal value integrals in the boundary element method, ASME J. Appl. Mech. 57 (1990) 906-915.

[23] M. Guiggiani, G. Krishnasamy, T.J. Rudolphi, F.J. Rizzo, A general algorithm for the numerical solution of hypersingular boundary integral equations, ASME J. Appl. Mech. 59 (1992) 604-614.

[24] M. Guiggiani, Hypersingular formulation for boundary stress evaluation, Engrg. Anal. Bound. Elem. 14 (1994) $169-179$.

[25] M. Guiggiani, Hypersingular boundary integral equations have an additional free term, Comp. Mech. 16 (1995) $245-248$.

[26] A. Frangi, M. Guiggiani, Boundary element analysis of Kirchhoff plates with direct evaluation of hypersingular integrals, Int. J. Num. Methods Engrg. 46 (1999) 1845-1863.

[27] A. Frangi, M. Guiggiani, A direct approach for boundary integral equations with high-order singularities, Int. J. Num. Methods Engrg. 49 (2000) 871-898.

[28] M. Guiggiani, Formulation and numerical treatment of boundary integral equations with hypersingular kernels, in: V. Sladek, J. Sladek (Eds.), Singular Integrals in Boundary Element Methods, Comp. Mech. Publ, Southampton, 1998, pp. 85-124, Chapter 3.

[29] P. Parreira, M. Guiggiani, On the implementation of the Galerkin approach in the boundary element method, Comp. Struct. 33 (1989) 269-279.

[30] A. Frangi, M. Guiggiani, Free terms and compatibility conditions for 3D hypersingular boundary integral equations, ZAMM 81 (2001) 651-664. 